\title{
Finite Range Decomposition of Gaussian Processes*
}

\author{
David C. Brydges, ${ }^{1}$ G. Guadagni, ${ }^{2}$ and P. K. Mitter $^{3}$
}

Received March 4, 2003; accepted June 2, 2003

\begin{abstract}
Let $\Delta$ be the finite difference Laplacian associated to the lattice $\mathbf{Z}^{d}$. For dimension $d \geqslant 3, a \geqslant 0$, and $L$ a sufficiently large positive dyadic integer, we prove that the integral kernel of the resolvent $G^{a}:=(a-\Delta)^{-1}$ can be decomposed as an infinite sum of positive semi-definite functions $V_{n}$ of finite range, $V_{n}(x-y)=0$ for $|x-y| \geqslant O(L)^{n}$. Equivalently, the Gaussian process on the lattice with covariance $G^{a}$ admits a decomposition into independent Gaussian processes with finite range covariances. For $a=0, V_{n}$ has a limiting scaling form $L^{-n(d-2)} \Gamma_{c, *}\left(\frac{x-y}{L^{n}}\right)$ as $n \rightarrow \infty$. As a corollary, such decompositions also exist for fractional powers $(-\Delta)^{-\alpha / 2}, 0<\alpha \leqslant 2$. The results of this paper give an alternative to the block spin renormalization group on the lattice.
\end{abstract}

KEY WORDS: Gaussian processes; finite range decomposition; lattice; renormalization group; Lévy processes.

\section{INTRODUCTION}

A smooth Gaussian process $\zeta(x)$ on $\mathbf{R}^{d}$ with the property that the expectation $E \zeta(x) \zeta(y)=0$ when $|x-y| \geqslant L$ will be said to have finite range $L$. What is the class of Gaussian processes $\phi$ that can be expressed as a sum $\phi=\sum_{j} \zeta_{j}$ of independent finite range processes with ranges $\sim L^{j}$ for some $L$ ? Let us call such processes finite range decomposable.

We can reformulate this in terms of the covariance: a Gaussian process $\phi$ is finite range decomposable if the covariance $C(x, y):=E \phi(x) \phi(y)$ can be written as a sum $C=\sum_{j} V_{j}$ where each $V_{j}(x, y)$ is positive semi-definite

\footnotetext{
* In Honour of G. Jona-Lasinio.

${ }^{1}$ The University of British Columbia (Laboratoire Associé au CNRS, UMR 5825), 1984 Mathematics Road, Vancouver, British Columbia, Canada V6T 1Z2.

${ }^{2}$ Department of Mathematics, University of Virginia, P.O. Box 400137, Charlottesville, Virginia 22904-4137.

${ }^{3}$ Laboratoire de Physique Mathématique, Université Montpellier 2, Place E. Bataillon, Case 070, 34095 Montpellier Cedex 05, France; e-mail: mitter@lpm.univ-montp2.fr
} 
and has finite range $\sim L^{j}$. In this form the question has already received a partial answer in the study of ground states for many-body Hamiltonians. In particular, in ref. $10 \mathrm{Hainzl}$ and Seiringer discuss this background and consider the decomposition

$$
V(x)=\int_{0}^{\infty} d r g(r) \chi_{r / 2} * \chi_{r / 2}(x)
$$

of a radial function $V(x)$ as a weighted integral of tent functions $\chi_{r / 2} * \chi_{r / 2}(x)$, where $\chi_{r / 2}$ is the indicator function of the ball of radius $r / 2$. An explicit formula for $g$ in terms of $V$ is derived. For example, in three dimensions,

$$
g(r)=-\frac{2}{\pi}\left(V^{\prime \prime}(r) / r\right)^{\prime}
$$

so necessary and sufficient conditions for $g \geqslant 0$ in terms of $V$ are readily formulated. In particular Coulomb and Yukawa potentials in three dimensions have decompositions with nonnegative $g$.

This is relevant to our question because the tent function is positive semi-definite and therefore, when $g(r) \geqslant 0$ and $I$ is an interval $[a, b)$,

$$
V_{I}(x):=\int_{I} d r g(r) \chi_{r / 2} * \chi_{r / 2}(x)
$$

is also positive semi-definite. By breaking up the range of the $r$ integration in (1.1) into a disjoint union of intervals, $I_{j}:=\left[L^{j}, L^{j+1}\right), j \in \mathbf{Z}$, we have $V=\sum V_{I_{j}}$ and there is a corresponding finite range decomposition $\phi=\sum_{j} \zeta_{j}$ when $\phi$ is the Gaussian process with covariance $V(x-y)$ with $g(r) \geqslant 0$ and $\zeta_{j}$ has covariance $V_{I_{j}}$.

These decompositions are not the final answer to our question, because we are also interested in kernels defined on the lattice $\mathbf{Z}^{d}$ and furthermore one may get a wider class by not insisting on decompositions based on tent functions. For lattices or the continuum we have preliminary results that suggest that resolvents of quite general elliptic operators and fractional inverse powers of elliptic operators are candidates for such decompositions.

Our interest in this question is rooted in the Renormalization Group (RG). In quantum field theory and other contexts the RG is a method to calculate the expectation $E \mathscr{Z}$ of a functional $\mathscr{Z}=\mathscr{Z}(\phi)$ of a Gaussian field $\phi$. One decomposes $\phi=\sum_{j \geqslant 1} \zeta_{j}$ as a sum of independent Gaussian fields $\zeta_{j}$ and integrates out each $\zeta_{j}$ one at a time. Let $E_{j}$ be the expectation that integrates out $\zeta_{j}$. Then the $\mathrm{RG}$ is the sequence of maps 
$\mathscr{Z}_{j} \mapsto \mathscr{Z}_{j+1}:=E_{j+1} \mathscr{Z}_{j} . E \mathscr{Z}$ is obtained from $E \mathscr{Z}=\lim \mathscr{Z}_{j}$, starting with $\mathscr{Z}_{0}:=\mathscr{Z}$. [These ideas are explained further in Section 4]. The point is to choose the decomposition to have special properties so that each expectation $E_{j}$ is more amenable to analysis than the whole expectation $E$ and furthermore so that the map $\mathscr{Z}_{j} \mapsto \mathscr{Z}_{j+1}$ can be analysed within the context of dynamical systems. In particular the $R G$ is very informative when the limiting map is autonomous, up to a scaling. This is possible when the covariances $V_{j}$ of $\zeta_{j}$ are becoming self-similar. This means that there should exist a dimension $[\phi]$ such that $V_{j}(x, y)=: L^{-2 j[\phi]} \Gamma_{j}\left(L^{-j} x, L^{-j} y\right)$ defines a scaled covariance $\Gamma_{j}$ which tends to a limit as $j \rightarrow \infty$.

When the covariances $V_{j}$ are finite range, the map $\mathscr{Z}_{j} \mapsto \mathscr{Z}_{j+1}$ can be studied by using the independence of $\zeta_{j}(x)$ and $\zeta_{j}(y)$ for $|x-y| \geqslant L^{j}$. We amplify on this remark at the end of this introduction. In some ways, the use of these finite range covariances gives the simplest framework that goes beyond the hierarchical models. This program is also close to the technique that was invented by Fröhlich and Spencer in ref. 6 to study the Kosterlitz-Thouless transition, but it is in principle more precise and more robust.

Let $\Delta$ be the finite difference Laplacian associated to the lattice $\mathbf{Z}^{d}$. We will consider decompositions for the kernel $G^{a}(x-y)$ of the resolvent $(a-\Delta)^{-1}$ and we will also consider the Green's function of a stable Lévy process which is the kernel $C(x-y)$ of $(-\Delta)^{-\alpha / 2}$ where $0<\alpha<2$. The mass parameter $a \in[0, \infty)$. We state our results for dimensions $d \geqslant 3$ because lower dimensions require extra discussions for the case $a=0$, but the basic construction is valid in lower dimensions as well. $L$ is a parameter of the form $2^{p}$. $p$ can be any sufficiently large integer. There are many technical results in this paper so we have summarised the main points in the following theorem which is a combination of results from the theorems in the rest of the paper.

Theorem 1.1. For $n=0,1,2, \ldots$, and all $a \geqslant 0$, there are positive semi-definite functions $\Gamma_{n}^{a}(x)$ defined for $x \in\left(L^{-n} \mathbf{Z}\right)^{d}$ such that

1. $G^{a}(x-y)=\sum L^{-2 n[\phi]} \Gamma_{n}^{L^{2 n} a}\left(\frac{x-y}{L^{n}}\right)$ with $[\phi]=(d-2) / 2$

2. $\Gamma_{n}^{a}(x)=0$ for $|x| \geqslant 6 L$

3. $\left|\hat{\Gamma}_{n}^{a}(p)\right| \leqslant c_{k, L}(1+|p|)^{-k}$ for $p \in\left[-L^{n} \pi, L^{n} \pi\right]^{d}$

4. $\hat{\Gamma}_{c, *}^{a}(p):=\lim _{n \rightarrow \infty} \hat{\Gamma}_{n}^{a}(p)$ exists pointwise in $p$

5. Fix a positive integer $l$ and let $\epsilon=L^{-l}$. Then $\Gamma_{c, *}^{a}=\lim _{n \rightarrow \infty} \Gamma_{n}^{a}$ exists in $L^{\infty}\left((\epsilon \mathbf{Z})^{d}\right)$. Furthermore the $L^{\infty}$ limit of any multiple lattice 
derivative of $\Gamma_{n}^{a}$ also exists and is the corresponding continuum derivative of $\Gamma_{c, *}^{a}$

6. Analogous statements hold for $C(x-y)$, but with $[\phi]=(d-\alpha) / 2$.

The multiscale expansion in (1) above is obtained in (3.31) of Section 3, and the finite range property (2) is given in Lemma 3.2. The bound in (3) follows from Theorem 5.5 of Section 5. (4) above is obtained in Section 6 in the course of proving Theorem 6.1, see (6.24). (5) above is part of Theorem 6.1. Turning to (6) above, the finite range decomposition of $C$ is obtained in Section 4 (see (4.1), (4.2) et seq.). A uniform bound on Lévy fluctuation covariances is supplied in Section 5 (see Corollary 5.6). The statement analogous to (5) above is part of Corollary 6.2 of Section 6.

Let us call the Gaussian fields in the decomposition fluctuation fields. Other (wavelet) decompositions were developed in the context of the Block Spin Renormalization Group of Kadanoff and Wilson by Gawedzki and Kupiainen, ${ }^{(7,8)}$ as well as, in related work, by Balaban. ${ }^{(3,4)}$ Although the Gawedzki-Kupiainen fluctuation fields are not finite range, they have their own advantages: notably they are independent lattice fields, determined by random variables defined on increasingly coarse lattices of spacing $L^{n}$. Our decomposition achieves this only in the weaker sense that, with high probability, $\zeta_{n}(x) \approx \zeta_{n}(y)$ for $|x-y| \ll L^{n}$, but $\zeta_{n}$ retains low probability variations on all smaller scales. This is a price paid for retaining translation invariance on small scales.

In Section 3 the aforementioned finite range decompositions are obtained. The rescaled fluctuation covariances live on finer and finer lattices, but all have the same finite range. In Section 4 probabilistic aspects of our construction are discussed and the finite range decomposition of the Lévy Greens function is obtained. We are interested in the Lévy Greens function $(-\Delta)^{-\alpha / 2}$ because varying the parameter $\alpha$ affects the scaling of the associated field and gives insight into the dynamical system $\mathscr{Z}_{j} \mapsto \mathscr{Z}_{j+1}$. Moreover it is of intrinsic interest in various problems in probability theory. For example we may wish to study critical properties of self avoiding Lévy walks by renormalization group methods. We also discuss in this section renormalization group transformations based on the above finite range decompositions.

Section 5 is devoted to bounds. Here our main result is Theorem 5.5. This theorem states that every member of the sequence of rescaled fluctuation covariances is uniformly bounded in lattice Sobolev norms of arbitrarily high degree. The bound is independent of the lattice spacing. Finally in Section 6 we prove that the sequence converges in Sobolev norms in a precise sense to its continuum limit which is appropriately identified. The continuum limit is smooth. This is the content of our main Theorem 6.1. 
We conclude with a brief indication of the role of the finite range property in the analysis of $\mathscr{Z}_{j} \mapsto \mathscr{Z}_{j+1}$. Consider $\mathscr{Z}_{0} \mapsto \mathscr{Z}_{1}$ when

$$
\mathscr{Z}_{0}=\mathscr{Z}_{0}\left(\Lambda, \phi_{0}\right):=\prod_{x \in \Lambda} e^{-\lambda \phi_{0}^{4}(x)}
$$

where $\Lambda \subset \mathbf{Z}^{d}$ is a large box shaped subset of lattice points which is a disjoint union of some standard cube shaped subsets of lattice points $\Delta \subset \mathbf{Z}^{d}$ of side greater than the range of the first field $\zeta_{1}$ in a finite range decomposition $\phi_{0}=\sum_{j \geqslant 1} \zeta_{j}$. Let $\phi_{1}:=\sum_{j \geqslant 2} \zeta_{j}, X \subset \mathbf{Z}^{d}$ and

$$
\tilde{\mathscr{Z}}_{1}(X)=\prod_{x \in X} e^{-\lambda \phi_{1}^{4}(x)}
$$

Note that $\tilde{\mathscr{Z}}_{1}(X)$ is independent of $\zeta_{1}$. Then

$$
\mathscr{Z}_{1}(\Lambda)=E_{1} \prod_{\Delta \subset \Lambda} \mathscr{Z}_{0}(\Delta)=E_{1} \prod_{\Delta \subset \Lambda}\left(\mathscr{Z}_{0}(\Delta)-\tilde{\mathscr{Z}}_{1}(\Delta)+\tilde{\mathscr{Z}}_{1}(\Delta)\right)
$$

Write $\delta \mathscr{Z}_{0}(\Delta):=\mathscr{Z}_{0}(\Delta)-\tilde{\mathscr{Z}}_{1}(\Delta)$. The product expands into a sum over $X$ of terms

$$
\prod_{\Delta \notin \subset X} \tilde{\mathscr{Z}}_{1}(\Delta) \prod_{\Delta \subset X} \delta \mathscr{Z}_{0}(\Delta)
$$

In other words, $X$ labels the factors where $\delta \tilde{\mathscr{Z}}_{0}$ is selected. We can partition $X$ into disjoint connected components $X_{1}, \ldots, X_{M}$, where $X_{i}$ is connected when the cubes $\Delta$ in $X_{i}$ are such that one can pass between any pair of cubes by a path whose steps are nearest neighbour cubes in $X_{i}$. Let

$$
K_{0}\left(X_{i}\right):=\prod_{\Delta \subset X_{i}} \delta \mathscr{Z}_{0}(\Delta)
$$

Then

$$
\mathscr{Z}_{1}(\Lambda)=\sum \frac{1}{M !} \sum_{X_{1}, \ldots, X_{M}} \tilde{\mathscr{Z}}_{1}\left(X_{0}\right) E_{1} \prod_{i} K_{0}\left(X_{i}\right)
$$

where the connected sets $X_{j}$ are disjoint and $X_{0}:=\Lambda \backslash \bigcup_{i \geqslant 1} X_{i}$. Notice that $\tilde{\mathscr{Z}}_{1}$ can be and has been moved outside the expectation since it is independent of $\zeta_{1}$. Now comes the key point: the sets $X_{j}$ are connected unions of nearest neighbour cubes of side length greater than the range of $\zeta_{1}$. Since 
they are disjoint they are separated by a distance greater than the range of $\zeta_{1}$. Therefore, by the finite range property,

$$
\mathscr{Z}_{1}(\Lambda)=\sum \frac{1}{M !} \sum_{X_{1}, \ldots, X_{M}} \tilde{\mathscr{Z}}_{1}\left(X_{0}\right) \prod_{i} E_{1} K_{0}\left(X_{i}\right)
$$

The perturbation on a large volume $\Lambda$ has been reduced to local calculations $E_{1} K_{0}\left(X_{i}\right)$ and the standard but heavy machinery of cluster expansions is being replaced by independence and geometry.

Unlike $\mathscr{Z}_{0}(\Lambda)$ the image functional $\mathscr{Z}_{1}(\Lambda)$ no longer factors into contributions from boxes, which is the great simplification of hierarchical models, but there is still a large part $X_{0}$ of $\Lambda$ where this property is retained. For the next RG map one proves that the more general form (1.2) is stable in the sense that $\mathscr{Z}_{2}$ can also be written in the same form but with a different $K_{1}$ and with larger cubes $L \Delta$. The program is then to prove that $E_{j} K_{j-1}(X)$ gives very little weight to connected sets $X$ which are unions of many boxes $L^{j-1} \Delta$. This can be facilitated by making a better choice of $\tilde{\mathscr{Z}}_{1}$, since the derivation is valid for other choices of $\tilde{\mathscr{Z}}_{1}$. In particular, one can replace $\lambda$ in $\tilde{\mathscr{Z}}_{1}$ by some other value $\lambda_{1}$ chosen to minimise $E_{1} K_{0}$. This idea leads to a flow of the coupling constant $\lambda \rightarrow \lambda_{1}$.

The first use of finite range covariances was in ref. 11. Reference 5 is another appearance of finite range covariances.

\section{PRELIMINARIES}

Throughout we will assume that $d \geqslant 3$. Let $L$ be a large integer power of 2. Define $\varepsilon_{n}=L^{-n}$. We will be working on a sequence of lattices $\left(\varepsilon_{n} \mathbf{Z}\right)^{d} \subset \mathbf{R}^{d},\left(\varepsilon_{n} \mathbf{Z}\right)^{d} \subset\left(\varepsilon_{n+1} \mathbf{Z}\right)^{d}$, with $n=0,1,2, \ldots$ and eventually passing to $\mathbf{R}^{d}$. $\left(\varepsilon_{n} \mathbf{Z}\right)^{d}$ is equipped with the discrete topology. The measurable sets are subsets of points and the measure $d z$ on $\left(\varepsilon_{n} \mathbf{Z}\right)^{d}$ is defined by

$$
\int_{\left(\varepsilon_{n} \mathbf{Z}\right)^{d}} d z f(z)=\varepsilon_{n}^{d} \sum_{z \in\left(\varepsilon_{n} \mathbf{Z}\right)^{d}} f(z)
$$

We endow $\mathbf{R}^{d}$ with the distance function

$$
|x-y|=\max _{1 \leqslant j \leqslant d}\left|x_{j}-y_{j}\right|
$$

Let

$$
U(R)=\left(-\frac{R}{2}, \frac{R}{2}\right)^{d} \subset \mathbf{R}^{d}
$$


be an open cube of edge length $R$. Define

$$
U_{\varepsilon_{n}}(R)=U(R) \cap\left(\varepsilon_{n} \mathbf{Z}\right)^{d}
$$

and its boundary

$$
\partial U_{\varepsilon_{n}}=\left\{y \notin U_{\varepsilon_{n}}:|x-y|=\varepsilon_{n}, \text { some } x \in U_{\varepsilon_{n}}\right\}
$$

The distance function $|$.$| is that induced from \mathbf{R}^{d}$. We denote by $\bar{U}_{\varepsilon_{n}}=U_{\varepsilon_{n}} \cup \partial U_{\varepsilon_{n}}$ the closure of $U_{\varepsilon_{n}}$. The lattice Laplacian $\Delta_{\varepsilon_{n}}$ is defined by the quadratic form

$$
\left(f,-\Delta_{\varepsilon_{n}} f\right)_{\left.L^{2}\left(\varepsilon_{n} Z\right)^{d}\right)}=\varepsilon_{n}^{d} \sum_{\langle x, y\rangle} \varepsilon_{n}^{-2}|f(x)-f(y)|^{2}
$$

where the sum runs over the nearest neighbour points in $\left(\varepsilon_{n} \mathbf{Z}\right)^{d}$. Let $\mathscr{S}=\left\{\hat{e}_{1}, \ldots, \hat{e}_{d}\right\}$ be the set of standard unit basis vectors in $\mathbf{Z}^{d}$. For any such lattice unit vector $\underline{e} \in \mathscr{S}$ define forward and backward lattice derivatives $\nabla_{ \pm \underline{e}}$ of a function in the direction $\underline{e}$ by

$$
\left(\nabla_{ \pm \underline{e}} f\right)(x)=\varepsilon_{n}^{-1}\left(f\left(x \pm \varepsilon_{n} \underline{e}\right)-f(x)\right)
$$

The backward derivative is defined so as to be the adjoint of the forward derivative. Then the definition (2.6) for the lattice Laplacian can be written as

$$
\left(f,-\Delta_{\varepsilon_{n}} f\right)_{L^{2}\left(\left(\varepsilon_{n} Z\right)^{d}\right)}=\varepsilon_{n}^{d} \sum_{x, \underline{e} \in \mathscr{S}}\left|\left(\nabla_{\underline{e}} f\right)(x)\right|^{2}
$$

The corresponding resolvent $G_{\varepsilon_{n}}^{a}$ with $a \geqslant 0$ is

$$
\begin{aligned}
G_{\varepsilon_{n}}^{a}(x-y) & =\left(-\Delta_{\varepsilon_{n}}+a\right)^{-1}(x-y) \\
& =\int_{\left[-\pi / \varepsilon_{n}, \pi / \varepsilon_{n}\right]^{d}} \frac{d^{d} p}{(2 \pi)^{d}} \frac{e^{i p .(x-y)}}{a-\hat{\Delta}_{\varepsilon_{n}}(p)}
\end{aligned}
$$

where

$$
\hat{\Delta}_{\varepsilon_{n}}(p)=2 \varepsilon_{n}^{-2} \sum_{\mu=1}^{d}\left(\cos \left(\varepsilon_{n} p_{\mu}\right)-1\right)
$$

\section{MULTISCALE DECOMPOSITION OF THE RESOLVENT}

We say that a function $f(x, y)$ has finite range $R$ if

$$
f(x, y)=0 \quad \text { for } \quad|x-y| \geqslant R
$$


Consider the resolvent in $\mathbf{Z}^{d}$

$$
G^{a}(x-y)=(-\Delta+a)^{-1}(x-y)
$$

with $a \geqslant 0$. We will first develop a multiscale decomposition for the resolvent $G^{a}, a \geqslant 0$, into smooth finite range positive semi-definite functions.

As in (2.3) and (2.4), $U(L) \subset \mathbf{R}^{d}$ is an open cube of edge length $L$ in $\mathbf{R}^{d}$ and $U_{\varepsilon}(L)=U(L) \cap(\varepsilon \mathbf{Z})^{d}$ the induced cube in $(\varepsilon \mathbf{Z})^{d}$ with $\partial U_{\varepsilon}(L)$ its boundary. Assume that the cube is centered at the origin. We will suppress the argument $L$ when there is no risk of confusion.

On the lattice there is no need to distinguish functions from measures. Nevertheless we use measures in cases where the associated continuum object is a measure. A case in point is the lattice Poisson kernel $\mathscr{P}_{U_{\varepsilon}}^{a}(x, d u)$, which by definition is the measure supported on $\partial U_{\varepsilon}$ such that

$$
h(x)=\mathscr{P}_{U_{\varepsilon}}^{a}(x, f):=\int \mathscr{P}_{U_{\varepsilon}}^{a}(x, d u) f(u)
$$

is the unique solution to the boundary value problem

$$
\begin{aligned}
\left(-\Delta_{\varepsilon}+a\right) h(x) & =0: x \in U_{\varepsilon} \\
h(x) & =f(x): x \in \partial U_{\varepsilon}
\end{aligned}
$$

where $f: \partial U_{\varepsilon} \rightarrow \mathbf{R}$. Existence and uniqueness are easily proved since $h$ solves a finite dimensional set of linear equations. Note that because $a \geqslant 0$ a solution $h(x)$ satisfies the weak maximum principle. In Section 4 we will see an explicit construction of which shows that $\mathscr{P}_{U_{\varepsilon}}^{a}$ is a defective probability measure. Defective means that the mass is at most one. (For $a=0$ and $f=1, h \equiv 1$ which implies $\mathscr{P}_{U_{\varepsilon}}^{a}(x, 1)$ is a probability measure). We will say $h$ is $a$-harmonic in $X_{\varepsilon}$ if $h$ solves $\left(-\Delta_{\varepsilon}+a\right) h(x)=0$ in $X \cap(\varepsilon Z)^{d}$.

Let $g(x)$ be a rotationally invariant non-negative $C^{\infty}\left(\mathbf{R}^{d}\right)$ function of compact support such that

$$
g(x)=0:|x| \geqslant \frac{L}{4}
$$

with the normalization

$$
\int_{\mathbf{R}^{d}} d x g(x)=1
$$


We restrict $g(x)$ to the lattice $(\varepsilon \mathbf{Z})^{d}$ and choose the normalization constant $c_{\varepsilon}$ such that

$$
\int_{(\varepsilon \mathbf{Z})^{d}} d x c_{\varepsilon} g(x)=1
$$

Since $g(x)$ is a continuous function of compact support Riemann sums converge. Hence $c_{\varepsilon}$ is a continuous function of $\varepsilon$ on the compact set $0 \leqslant \varepsilon \leqslant 1$ and thus uniformly bounded. Moreover $c_{\varepsilon} \rightarrow 1$ as $\varepsilon \rightarrow 0$.

Now comes the main idea. The point of the function $g$ is to avoid needing detailed knowledge of the Poisson kernel for the lattice. We are about to use the Poisson kernel to define an averaging operator that leaves a-harmonic functions unchanged. This property leads to the finite range property in Lemma 3.1. It is relatively easy to prove that our averaging operator is smoothing (uniformly in lattice spacing) because when checking differentiability, derivatives either fall on $g$ which is smooth by choice or on the $x$ argument of $\mathscr{P}_{U_{\varepsilon}}^{a}(x, d u)$ with $x$ forced to be away from the boundary $\partial U_{\varepsilon}$ so that the easy part of standard elliptic techniques is sufficient to prove smoothness uniformly in the lattice spacing.

Given a function $f:(\varepsilon \mathbf{Z})^{d} \rightarrow \mathbf{R}$ we define the averaging map:

$$
f \rightarrow A_{\varepsilon}^{a}(L) f
$$

where

$$
\left(A_{\varepsilon}^{a}(L) f\right)(x)=\int_{(\varepsilon \mathbf{Z})^{d}} d z c_{\varepsilon} g(z-x) \int \mathscr{P}_{U_{\varepsilon}(L)}^{a}(x-z, d u) f(u+z)
$$

Note that this can also be written as

$$
\left(A_{\varepsilon}^{a}(L) f\right)(x)=\int_{(\varepsilon Z)^{d}} d z c_{\varepsilon} g(z-x) \mathscr{P}_{U_{\varepsilon}(L, z)}^{a}(x, f)
$$

where $U_{\varepsilon}(L, z)$ is the translate of the cube $U_{\varepsilon}(L)$ so that its center is now $z$. In summing over the translates we have put in the smooth function $g$, and not the delta function, because we will need to take derivatives with respect to $x$ and this is hard to do if $x$ is the center of the cube.

Now this integration over all translates makes $A_{\varepsilon}^{a}(L)$ translation invariant. Translation invariance plays an essential role in the proof of positive semi-definiteness of the fluctuation covariance constructed below, 
(see Lemma 3.1). Proof: For $b \in(\varepsilon \mathbf{Z})^{d}$, let $f_{b}(x)=f(x-b)$. By change of variables $z \rightarrow z-b$ in (3.8)

$$
\left(A_{\varepsilon}^{a}(L) f\right)(x-b)=\left(A_{\varepsilon}^{a}(L) f_{b}\right)(x)
$$

From (3.8) we see that for every fixed $x,\left(A_{\varepsilon}^{a}(L) f\right)(x)$ defines a bounded, positive linear functional on $C_{0}\left((\varepsilon \mathbf{Z})^{d}\right)$, the space of functions of compact support on $(\varepsilon \mathbf{Z})^{d}$. We have

$$
\left|\left(A_{\varepsilon}^{a}(L) f\right)(x)\right| \leqslant\|f\|_{\infty}
$$

so that the norm of this linear functional is $\leqslant 1$. This gives a family of defective probability measures $A_{\varepsilon}^{a}(L)(x, d u)$ on $(\varepsilon \mathbf{Z})^{d}$

$$
\left(A_{\varepsilon}^{a}(L) f\right)(x)=\int_{(\varepsilon \mathbf{Z})^{d}} A_{\varepsilon}^{a}(L)(x, d u) f(u)
$$

The Fourier transform of this measure

$$
\hat{A}_{\varepsilon}^{a}(p)=\int_{(\varepsilon \mathrm{Z})^{d}} d u A_{\varepsilon}^{a}(L)(0, d u) e^{-i p \cdot u}
$$

satisfies

$$
\left|\hat{A}_{\varepsilon}^{a}(p)\right| \leqslant \int_{(\varepsilon \mathbf{Z})^{d}} d u A_{\varepsilon}^{a}(L)(0, d u) \leqslant 1
$$

Define the fluctuation covariance

$$
\Gamma_{\varepsilon}^{a}(x-y)=G_{\varepsilon}^{a}(x-y)-\left(A_{\varepsilon}^{a}(L) G_{\varepsilon}^{a} A_{\varepsilon}^{a}(L)^{*}\right)(x-y)
$$

where by definition

$\left(A_{\varepsilon}^{a}(L) G_{\varepsilon}^{a} A_{\varepsilon}^{a}(L)^{*}\right)(x-y)=\iint A_{\varepsilon}^{a}(L)(x, d u) G^{a}(u-v) A_{\varepsilon}^{a}(L)(y, d v)$

The latter is the analogue of the block spin covariance in statistical mechanics. ${ }^{(9)}$

Lemma 3.1. $\Gamma_{\varepsilon}^{a}$ and $A_{\varepsilon}^{a}(L) G_{\varepsilon}^{a} A_{\varepsilon}^{a}(L)^{*}$ are positive semi-definite. $\Gamma_{\varepsilon}^{a}$ has finite range,

$$
\Gamma_{\varepsilon}^{a}(x-y)=0:|x-y| \geqslant 3 L
$$


and the Fourier transform $\hat{\Gamma}_{\varepsilon}^{a}(p)$ is continuous in $p$ including at $p=0$, uniformly in $\varepsilon$.

Proof. First we prove the finite range property. By the definition of the Poisson kernel, if $f$ is $a$-harmonic in $x+U_{\varepsilon}$, then $\mathscr{P}_{U_{\varepsilon}}^{a}(x, f)=f(x)$. Since $c_{\varepsilon} g$ in the definition of $A_{\varepsilon}^{a}(L)$ was chosen to be a probability density with support in $U_{\varepsilon}(L / 4)$, for $f a$-harmonic in $x+U_{\varepsilon}(5 L / 4)$,

$$
\begin{aligned}
\left(A_{\varepsilon}^{a}(L) f\right)(x) & =\int_{(\varepsilon \mathrm{Z})^{d}} d z c_{\varepsilon} g(z-x) \mathscr{P}_{U_{\varepsilon}(L, z)}^{a}(x, f) \\
& =\int_{(\varepsilon \mathrm{Z})^{d}} d z c_{\varepsilon} g(z-x) f(x)=f(x)
\end{aligned}
$$

When $|x-y| \geqslant 3 L, x+U_{\varepsilon}(5 L / 4)$, and $y+U_{\varepsilon}(5 L / 4)$ are disjoint. Therefore $G_{\varepsilon}^{a}(u-v)$ is $a$-harmonic in each argument in the appropriate region and therefore

$$
\left(A_{\varepsilon}^{a}(L) G_{\varepsilon}^{a} A_{\varepsilon}^{a}(L)^{*}\right)(x-y)=G_{\varepsilon}^{a}(x-y)
$$

which proves (3.15). Now we prove positive definiteness. By translation invariance, we can take the Fourier transform of $\Gamma_{\varepsilon}^{a}(x-y)$ to get

$$
\hat{\Gamma}_{\varepsilon}^{a}(p)=\left(1-\left|\hat{A}_{\varepsilon}^{a}(p)\right|^{2}\right) \hat{G}_{\varepsilon}^{a}(p)
$$

Now $\hat{G}_{\varepsilon}^{a}(p) \geqslant 0$ and $\left|\hat{A}_{\varepsilon}^{a}(p)\right| \leqslant 1$ where we have used (3.12). Hence $\hat{\Gamma}_{\varepsilon}^{a}(p) \geqslant 0$. This proves positive definiteness of $\Gamma_{\varepsilon}^{a}$. The positive definiteness of $A_{\varepsilon}^{a} G_{\varepsilon}^{a} A_{\varepsilon}^{a *}$ is obvious.

Continuity of $\hat{\Gamma}_{\varepsilon}^{a}(p)$ : By (2.10),

$$
\hat{G}_{\varepsilon}^{a}(p)=\frac{1}{a-\hat{\Delta}_{\varepsilon_{n}}(p)}
$$

is continuous if $a>0$ or $p \neq 0$. If $a=0$ then (3.16) shows that the $p^{-2}$ singularity of $\hat{G}_{\varepsilon}^{a}(p)$ is cancelled by

$$
\left|1-\hat{A}_{\varepsilon}^{a}(p)\right|=\left|\hat{A}_{\varepsilon}^{a}(0)-\hat{A}_{\varepsilon}^{a}(p)\right|=o\left(p^{2}\right)
$$

which holds because $p$ derivatives of $\hat{A}_{\varepsilon}^{a}(p)$ are moments for $A_{\varepsilon}^{a}$ and this is a defective probability measure of compact support which therefore has moments of all orders. 
Choose $\varepsilon=\varepsilon_{n-1}$ for $n \geqslant 1$ and write (3.13) in a rescaled form. It is easy to check that for $u, v \in\left(\varepsilon_{n-1} \mathbf{Z}\right)^{d}$ we have

$$
\begin{aligned}
G_{\varepsilon_{n-1}}^{a}(u-v) & =L^{-(d-2)} G_{\varepsilon_{n}}^{L^{2} a}\left(\frac{u-v}{L}\right) \\
\mathscr{P}_{U_{\varepsilon_{n-1}}(R, z)}^{a}(x, d u) & =\mathscr{P}_{U_{\varepsilon_{n}}\left(\frac{R}{L}, \frac{z}{L}\right)}^{L^{2} a}\left(\frac{x}{L}, \frac{d u}{L}\right)
\end{aligned}
$$

Define the sequence of functions $g_{n}$ on $\mathbf{R}^{d}$ by

$$
g_{n}(z)=L^{n d} g\left(L^{n} z\right)
$$

where $g$ is the function introduced earlier (see (3.5), (3.6)) and observe that because of the normalization (3.6), the function $g_{n}$ is also normalized :

$$
\int_{\mathbf{R}^{d}} d z g_{n}(z)=1
$$

Moreover, from the support property of $g$, we have that

$$
g_{n}(x)=0:|x| \geqslant \frac{1}{4 L^{n-1}}
$$

Let

$$
R_{m}=L^{-(m-1)}
$$

As in (3.11) we have in $\left(\varepsilon_{n} \mathbf{Z}\right)^{d}$ for $n \geqslant m \geqslant 0$ the measure $A_{\varepsilon_{n}, m}^{a}\left(R_{m}\right)(x, d u)$ given by

$$
\int_{\left(\varepsilon_{n} \mathbf{Z}\right)^{d}} d u A_{\varepsilon_{n}, m}^{a}\left(R_{m}\right)(x, d u) f(u)=\int_{\left(\varepsilon_{n} Z\right)^{d}} d z c_{\varepsilon_{n-m}} g_{m}(x-z) \mathscr{P}_{U_{\varepsilon}\left(R_{m}, z\right)}^{a}(x, f)
$$

Note that

$$
A_{\varepsilon_{n}, 0}^{a}=A_{\varepsilon_{n}}^{a}
$$

as defined earlier. Observe that

$$
\int_{\left(\varepsilon_{n} Z\right)^{d}} d z c_{\varepsilon_{n-m}} g_{m}(z)=\int_{\left(\varepsilon_{n-m} \mathrm{Z}\right)^{d}} d z c_{\varepsilon_{n-m}} g(z)=1
$$


from the definition of the constants $c_{\varepsilon_{n}}$ in (3.7). The definition (3.19) together with (3.23) and (3.18) imply the scaling relation

$$
A_{\varepsilon_{n-1}, m-1}^{a}\left(R_{m-1}\right)(x, d u)=A_{\varepsilon_{n}, m}^{L^{2} a}\left(R_{m}\right)\left(\frac{x}{L}, \frac{d u}{L}\right)
$$

Applying this to the righthand side of (3.13) we get for $n \geqslant 1$

$$
G_{\varepsilon_{n-1}}^{a}(x-y)=\Gamma_{\varepsilon_{n-1}}^{a}(x-y)+L^{-(d-2)}\left(A_{\varepsilon_{n}, 1}^{L^{2} a}(1) G_{\varepsilon_{n}}^{L^{2} a} A_{\varepsilon_{n}, 1}^{L^{2} a}(1)^{*}\right)\left(\frac{x-y}{L}\right)
$$

We can now iterate (3.27) starting with $n=1, n$-times using the same principle. Define for $n \geqslant 1$

$$
\mathscr{A}_{n}^{a}=\prod_{j=1}^{n} A_{\varepsilon_{n}, n+1-j}^{a}\left(L^{-(n-j)}\right)
$$

For $n=0$ we set

$$
\mathscr{A}_{0}^{a}=1
$$

We also define on the $\left(\varepsilon_{n} \mathbf{Z}\right)^{d}$ lattice

$$
\Gamma_{n}^{a}=\mathscr{A}_{n}^{a} \Gamma_{\varepsilon_{n}}^{a} \mathscr{A}_{n}^{a *}
$$

and

$$
\mathscr{G}_{n}^{a}=\mathscr{A}_{n}^{a} G_{\varepsilon_{n}}^{a} \mathscr{A}_{n}^{a *} .
$$

Then we have the multiscale decomposition for the resolvent

$$
G^{a}(x-y)=\sum_{j=0}^{n-1} L^{-j(d-2)} \Gamma_{j}^{L^{2 j} a}\left(\frac{x-y}{L^{j}}\right)+L^{-n(d-2)} \mathscr{G}_{n}^{L^{2 n} a}\left(\frac{x-y}{L^{n}}\right)
$$

which is valid for $a \geqslant 0$. The special case $a=0$ gives the multiscale decomposition for the massless Green's function

$$
G^{0}(x-y)=\sum_{j=0}^{n-1} L^{-j(d-2)} \Gamma_{j}^{0}\left(\frac{x-y}{L^{j}}\right)+L^{-n(d-2)} \mathscr{G}_{n}^{0}\left(\frac{x-y}{L^{n}}\right)
$$

Lemma 3.2. For all $n \geqslant 0$

$$
\Gamma_{n}^{a}(x-y)=0:|x-y| \geqslant 6 L
$$

Proof. $\quad \Gamma_{n}^{a}$ is a multiple convolution, so the range of $\Gamma_{n}^{a}$ is the sum of the ranges of the convolved functions. From the definition (3.23) of 
$A_{\varepsilon_{n}, m}^{a}(R)$, the support property (3.21) of $g_{m}$ and $\mathscr{P}_{U_{\varepsilon_{n}}}^{a}(R)(x, u)$ vanishes if $|u-z|>R$, we find that the range of $A_{\varepsilon_{n}, m}^{a}(R)$ is $R+\frac{\varepsilon_{\varepsilon_{1}}}{4 L^{m-1}}$. From the definition (3.28) of $\mathscr{A}_{n}^{a}$ the range of $\mathscr{A}_{n}^{a}$ is $\sum_{j=1}^{n} L^{-(n-j)}\left(1+\frac{1}{4 L}\right)$ which is less than 3 for $L$ large. By construction the range of $\Gamma_{\varepsilon_{n}}^{a}$ is less than $3 L$ by Lemma 3.1. The Lemma follows.

\section{PROBABILISTIC ASPECTS}

\section{Multiscale Decomposition for the Lévy Green's Function}

Let $x_{t}^{(\alpha)}, 0<\alpha<2$, be the stable Lévy process in $Z^{d}$, (stable in the sense that its scaling limit is stable). Note that $E_{x}\left(\left|x_{t}^{(\alpha)}\right|\right)<\infty$, provided $\alpha>1$. However in the following we will allow for the full admissable range $0<\alpha<2$. The Lévy Green's function $C$ is given by

$$
C(x-y)=(-\Delta)^{-\alpha / 2}(x-y)=\text { const } \int_{0}^{\infty} d a a^{-\alpha / 2} G^{a}(x-y)
$$

with $0<\alpha<2$. In this range it is easy to verify that the integral representation converges.

$C$ has a finite range multiscale decomposition, which we obtain by inserting the multiscale decomposition (3.31) for $G^{a}$ into the integral representation (4.1) of the Lévy Green's function $C$ to get

$$
\begin{aligned}
C(x-y)= & \sum_{j=0}^{n-1} L^{-j(d-2)} \text { const } \int_{0}^{\infty} d a a^{-\alpha / 2} \Gamma_{j}^{L^{2 j} a}\left(\frac{x-y}{L^{j}}\right) \\
& +L^{-n(d-2)} \text { const } \int_{0}^{\infty} d a a^{-\alpha / 2} \mathscr{G}_{n}^{L^{2 n} a}\left(\frac{x-y}{L^{n}}\right)
\end{aligned}
$$

After rescaling in $a$ in each term we get

$$
C(x-y)=\sum_{j=0}^{n-1} L^{-2 j[\phi]} \Gamma_{j}\left(\frac{x-y}{L^{j}}\right)+L^{-2 n[\phi]} C_{n}\left(\frac{x-y}{L^{n}}\right)
$$

where

$$
\begin{aligned}
\Gamma_{j} & =\text { const } \int_{0}^{\infty} d a a^{-\alpha / 2} \Gamma_{j}^{a} \\
C_{n} & =\text { const } \int_{0}^{\infty} d a a^{-\alpha / 2} \mathscr{G}_{n}^{a} \\
{[\phi] } & =\frac{d-\alpha}{2}
\end{aligned}
$$


Note that $[\phi]$ as defined above is the canonical dimension of the scalar Gaussian field $\phi$ distributed with covariance $C$. We have $[\phi]>0$, since $d \geqslant 3$ and $0<\alpha<2 . \Gamma_{j}$ and $C_{n}$ are well defined because of the bounds provided below (see Section 5, Corollary 5.6). Moreover by Lemma 3.2 and (4.3)

$$
\Gamma_{n}(x-y)=0:|x-y| \geqslant 6 L
$$

From Lemma 3.1 and (4.3), $C_{n}$ and $\Gamma_{n}$ are positive semi-definite and thus qualify as covariances of Gaussian measures denoted $\mu_{C_{n}}, \mu_{\Gamma_{n}}$. The multiscale decomposition (4.2) now gives rise to renormalization group transformations. From (4.2) we get for $x, y \in\left(\varepsilon_{n} \mathbf{Z}\right)^{d}$

$$
C_{n}(x-y)=\Gamma_{n}(x-y)+L^{-2[\phi]} C_{n+1}\left(\frac{x-y}{L}\right)
$$

and hence we have a sequence of RG transformations

$$
z_{n+1}(\phi)=\int d \mu_{\Gamma_{n}}(\zeta) z_{n}\left(\zeta+\phi_{L^{-1}}\right)
$$

where

$$
\phi_{L^{-1}}(x)=L^{-[\phi]} \phi\left(\frac{x}{L}\right)
$$

and

$$
\int d \mu_{C_{n}}(\phi) z_{n}(\phi)=\int d \mu_{C_{n+1}}(\phi) z_{n+1}(\phi)
$$

with $C_{0}=C$ given by (4.1).

\section{Poisson Kernel}

Let $x_{t}^{(n)}, t \geqslant 0$ be continuous time simple random walk with right continuous paths and state space $\left(\varepsilon_{n} \mathbf{Z}\right)^{d}$. The characteristic function is

$$
E\left(e^{i p \cdot x_{t}^{(n)}}\right)=e^{t{\hat{\Delta_{n}}}_{n}(p)}
$$

and the generator of the Markov process $x_{t}^{(n)}$ is the lattice Laplacian $\Delta_{\varepsilon_{n}}$. Note that the semigroup $e^{t \Delta_{\varepsilon_{n}}}$ is a contraction on $L^{\infty}\left(\left(\varepsilon_{n} \mathbf{Z}\right)^{d}\right)$. In the discrete topology the latter coincides with the space of bounded continuous functions. Hence $e^{t \Delta_{\varepsilon_{n}}}$ is a Feller semigoup so that $x_{t}^{(n)}$ is strong Markov with respect to stopping times $\tau$. 
Now let $x_{t}^{\varepsilon}$ be the above process in $(\varepsilon \mathbf{Z})^{d}$. Let $P_{x}$ the probability measure for the process conditioned to start at $x$. As in (2.3) and (2.4), $U(R)$ is an open cube of radius $R / 2$ in $\mathbf{R}^{d}, U_{\varepsilon}(R)$ the induced cube in $(\varepsilon \mathbf{Z})^{d}$, and the boundary $\partial U_{\varepsilon}(R)$ is defined as in (2.5). Let $\tau_{U_{\varepsilon}}$ be the first exit time from $U_{\varepsilon}$. Then $\tau_{U_{\varepsilon}}$ is also the first hitting time of $\partial U_{\varepsilon}$ from the interior. $E_{x}\left(\tau_{U_{\varepsilon}}\right)<\infty$ because $U_{\varepsilon}(R)$ is bounded, and hence $\tau_{U_{\varepsilon}}<\infty, P_{x}$ a.s.

Let $A$ be a subset of points in $\partial U_{\varepsilon}$. It is a standard result in probability that the measure $\mathscr{P}_{U_{\varepsilon}}^{a}(x, d y)$ defined on $\partial U_{\varepsilon}$ by

$$
\mathscr{P}_{U_{\varepsilon}}^{a}(x, A)=E_{x}\left(e^{-a \tau_{U_{\varepsilon}}} 1_{x_{\tau_{U_{\varepsilon}}}} \epsilon A\right)
$$

is the Poisson kernel we defined in (3.2).

Also, since $P_{x}\left(x_{\tau_{U_{\varepsilon}}} \in \partial U\right)=1$ the total mass is

$$
\mathscr{P}_{U_{\varepsilon}}^{a}\left(x, \partial U_{\varepsilon}\right)=E_{x}\left(e^{-a \tau_{U_{\varepsilon}}}\right) \leqslant 1
$$

The same construction works in $\mathbf{R}^{d}$ with $x_{t}^{\varepsilon}$ replaced by standard Brownian motion $x_{t}, U_{\varepsilon}(R)$ replaced by $U(R)$ and $A$ taken to be any Borel subset of $\partial U(R)$.

Let now $R=R_{m}=L^{-(m-1)}$, with $0 \leqslant m \leqslant n$. In Section 6 we will need an estimate for $1-\mathscr{P}_{U_{\varepsilon_{n}}}^{a}\left(x, \partial U_{\varepsilon_{n}}\right)$ where it is understood $U_{\varepsilon_{n}}=U_{\varepsilon_{n}}\left(R_{m}\right)$. Observe that $1-e^{-a \tau} \leqslant a \tau$ so

$$
0 \leqslant 1-\mathscr{P}_{U_{\varepsilon_{n}}}^{a}\left(x, \partial U_{\varepsilon_{n}}\right) \leqslant a E_{x}\left(\tau_{U_{\varepsilon}}\right)
$$

We can estimate the mean exit time as follows. Let $f$ be a smooth function in $\mathbf{R}^{d}$ bounded in a neighbourhood of $U(R)$. Then by the strong Markov property, for any $x \in U_{\varepsilon_{n}}\left(R_{m}\right)$,

$$
E_{x}\left(\int_{0}^{\tau_{U_{\varepsilon}}} d t\left(\Delta_{\varepsilon_{n}} f\right)\left(x_{t}\right)\right)=E_{x}\left(f\left(x_{\tau_{U_{\varepsilon}}}\right)\right)-f(x)
$$

We choose $x=0$ and $f(x)=|x|^{2}$ in $U\left(\frac{3}{2} R_{m}\right)$. Then by a simple computation in $U\left(R_{m}\right)$ we obtain $\Delta_{\varepsilon_{n}} f(x)=2 d$. Moreover as shown in Section 6, (6.14), $\partial U_{\varepsilon_{n}}\left(R_{m}\right) \subset \partial U(R)$. Hence we get the bound

$$
E_{x}\left(\tau_{U_{\varepsilon}}\right) \leqslant \frac{1}{2 d} E_{x}\left(x_{\tau_{U_{\varepsilon}}}^{2}\right) \leqslant \frac{R_{m}^{2}}{2}
$$

Thus we have proved the following. 
Lemma 4.1. Let $R_{m}=L^{-(m-1)}, 0 \leqslant m \leqslant n$ Then we have the bound

$$
0 \leqslant 1-\mathscr{P}_{U_{\varepsilon_{n}}\left(R_{m}\right)}^{a}\left(x, \partial U_{\varepsilon_{n}}\left(R_{m}\right)\right) \leqslant a \frac{R_{m}^{2}}{2}
$$

Now recall the definition of the measure $A_{\varepsilon_{n}, m}^{a}\left(R_{m}\right)(0, d u)$ on $\left(\varepsilon_{n} \mathbf{Z}\right)^{d}$ given in (3.23). $c_{\varepsilon_{n-m}} g_{m}(z)$ is a probability density in $(\varepsilon \mathbf{Z})^{d}$. Hence from (Lemma 4.1) we get

\section{Corollary 4.2.}

$$
0 \leqslant 1-\int_{\left(\varepsilon_{n} \mathrm{Z}\right)^{d}} A_{\varepsilon_{n}, m}^{a}\left(R_{m}\right)(0, d u) \leqslant a \frac{R_{m}^{2}}{2}
$$

\section{BOUNDS}

We will give uniform bounds on Fourier transforms and Sobolev norms of arbitrary high index. In Section 6 we will prove in the latter norms the convergence of the sequences $C_{n}, \Gamma_{n}$. The limiting covariances will thus turn out to be in $C^{p}, \forall p \geqslant 0$.

We recall the definition of the lattice derivative $\nabla_{\underline{e}}$ in (2.7). In particu$\operatorname{lar} \mathscr{S}=\left\{\hat{e}_{1}, \ldots, \hat{e}_{d}\right\}$ is the standard basis of unit vectors. For $\underline{e} \in \mathscr{S}, \nabla_{\underline{e}}$ is the forward partial derivative and for $-\underline{e} \in \mathscr{S}, \nabla_{\underline{e}}$ is the backward partial derivative. We define the $n$th lattice derivative

$$
\nabla_{\underline{e}_{1}, \ldots, \underline{e}_{n}}^{n}=\nabla_{\underline{e}_{1}} \cdots \nabla_{\underline{e}_{n}}
$$

Let $X$ be a connected open set in $\mathbf{R}^{d}$. We define

$$
X_{\varepsilon}=X \cap(\varepsilon \mathbf{Z})^{d}
$$

We now define the lattice Sobolev norm $\|\cdot\|_{H_{k}\left(X_{\varepsilon}\right)}$ of a function $f$ by

$$
\|f\|_{H_{k}\left(X_{\varepsilon}\right)}^{2}=\sum_{j=0}^{k} 2^{-j} \sum_{ \pm \underline{e}_{1}, \ldots, \pm \underline{e}_{j} \in \mathscr{S}} \int_{X_{\varepsilon}} d x\left|\nabla_{\underline{e}_{1}, \ldots, \underline{e}_{j}}^{j} f(x)\right|^{2}
$$

Lemma 5.1. Let $g$ be a $C^{\infty}\left(\mathbf{R}^{d}\right)$ function. Then for every $k \geqslant 0$ there exists a constant $C_{k}$ independent of $\varepsilon$ such that

$$
\|f g\|_{H_{k}\left(X_{\varepsilon}\right)} \leqslant C_{k}\|g\|_{C^{k}\left(\mathbf{R}^{d}\right)}\|f\|_{H_{k}\left(X_{\varepsilon}\right)}
$$


For every $k \geqslant 0$, and any $L_{1}$ function $g$,

$$
\|f * g\|_{\left.H_{k}\left((\varepsilon \mathbf{Z})^{d}\right)\right)} \leqslant\|g\|_{L_{1}\left((\varepsilon \mathbf{Z})^{d}\right)}\|f\|_{\left.H_{k}\left((\varepsilon \mathbf{Z})^{d}\right)\right)}
$$

Proof. To prove this we take the square of the norm on the left hand side and then use the $(\varepsilon \mathbf{Z})^{d}$ lattice modification of the Leibniz rule:

$$
\nabla_{\varepsilon, \underline{e}}(f g)=\left(\nabla_{\varepsilon, \underline{e}} f\right) g+f \nabla_{\varepsilon, \underline{e}} g+\varepsilon \nabla_{\varepsilon, \underline{e}} f \nabla_{\varepsilon, \underline{e}} g
$$

Derivatives on $g$ are bounded in the $C^{k}\left(\mathbf{R}^{d}\right)$ norm and all $\varepsilon$ dependent constants can be majorised by setting $\varepsilon=1$. This proves the first inequality. The second inequality, which is a form of Young's convolution inequality, is proved exactly as in the continuum.

In the following we will exploit a lattice version of elliptic regularity. Let $f$ be a bounded function in $(\varepsilon \mathbf{Z})^{d}$. Let $U(R) \subset \mathbf{R}^{\mathrm{d}}$ be an open cube centered at the origin and of edge length $R$. Let $U_{\varepsilon}(R)=U(R) \cap(\varepsilon \mathbf{Z})^{d}$. Let $\Omega \subset U\left(\frac{1}{4} R\right)$ be an open connected set. Then $\Omega_{\varepsilon} \subset U_{\varepsilon}\left(\frac{1}{4} R\right)$. Define

$$
h^{a}(x)=\mathscr{P}_{U_{\varepsilon}}^{a}(x, f)
$$

Recall from Section 2 (see (3.3), (3.4) et seq.) that $h^{a}(x)$ is the solution of the Dirichlet problem

$$
\begin{aligned}
\left(-\Delta_{\varepsilon}+a\right) h^{a}(x) & =0: x \in U_{\varepsilon} \\
h^{a}(u) & =f(u): u \in \partial U_{\varepsilon}
\end{aligned}
$$

and that the maximum principle holds, because $a \geqslant 0$. We have

Proposition 5.2 (Lattice Elliptic Regularity). With $\Omega_{\varepsilon}$ defined as above, and $\forall k \geqslant 0$

$$
\left\|h^{a}\right\|_{H_{k}\left(\Omega_{\varepsilon}\right)} \leqslant C_{R}(1+a)^{-\frac{1}{2}}\|f\|_{L^{\infty}\left((\varepsilon \mathbf{Z})^{d}\right)}
$$

Remark. This is well known in the continuum. For completeness we give a proof of the lattice version in Appendix A.

We choose $\varepsilon=\varepsilon_{n}$ and apply the proposition to

$$
\left(A_{\varepsilon_{n}, m=1}^{a}\left(L^{0}\right) f\right)\left(x^{\prime}\right):=\int_{\left(\varepsilon_{n} Z\right)^{d}} d z c_{\varepsilon_{n-1}} g_{1}\left(x^{\prime}-z\right) \mathscr{P}_{U_{\varepsilon_{n}}(1, z)}^{a_{n}}\left(x^{\prime}, f\right)
$$

which is the $j=n$ term in the product (3.28) defining $\mathscr{A}_{n}^{a}$. 
Corollary 5.3. Let $\Omega_{\varepsilon_{n}}$ be as in Proposition 5.2 with $R=1$. Then for every $k \geqslant 0$ and every $n \geqslant 1$ there exists a constant $C_{k, L}$ independent of $\varepsilon_{n}$ such that

$$
\begin{array}{r}
\left\|A_{\varepsilon_{n}, m=1}^{a}\left(L^{0}\right) f\right\|_{H_{k}\left(\Omega_{\varepsilon_{n}}\right)} \\
\leqslant C_{k, L}(1+a)^{-\frac{1}{2}}\|f\|_{L^{\infty}\left(\left(\varepsilon_{n} Z\right)^{d}\right)} \\
\left\|\nabla_{\underline{e}_{1}, \ldots, e_{k}}^{j} A_{\varepsilon_{n}, m=1}^{a}\left(L^{0}\right) f\right\|_{L_{\infty}\left(\left(\varepsilon_{n} Z\right)^{d}\right)} \leqslant C_{k, L}(1+a)^{-\frac{1}{2}}\|f\|_{L^{\infty}\left(\left(\varepsilon_{n} Z\right)^{d}\right)}
\end{array}
$$

Proof. The integral over $z$ in $(5.4,3.9)$ can be restricted to $U_{\varepsilon_{n}}(2)$ because the range of $g_{1}$ is $1 / 4$. Therefore

$$
\begin{aligned}
& \left\|A_{\varepsilon_{n}, m=1}^{a}\left(L^{0}\right)(\cdot, f)\right\|_{H_{k}\left(\Omega_{\varepsilon_{n}}\right)} \\
& \quad \leqslant \int_{U_{\varepsilon_{n}}(2)} d z c_{\varepsilon_{n-1}}\left\|g_{1}\right\|_{C^{k}\left(\mathbf{R}^{d}\right)}\left\|\mathscr{P}_{U_{\varepsilon_{n}}(1, z)}^{a_{n}}(\cdot, f)\right\|_{H_{k}\left(\Omega_{\varepsilon_{n}}\right)} \\
& \quad \leqslant c_{k, L}(1+a)^{-\frac{1}{2}}\|f\|_{L^{\infty}\left(\left(\varepsilon_{n} \mathbf{Z}\right)^{d}\right)}
\end{aligned}
$$

We have used the first inequality of Lemma 5.1, absorbing the $C^{k}\left(\mathbf{R}^{d}\right)$ norm of $g_{1}$ in the constant since $g$ has been fixed once for all, and then using Proposition 5.2.

To prove (5.5): By the embedding of high degree Sobolev space into $L_{\infty}$, (Lemma B.1), reviewed in Appendix B, we pass from (5.4) to

$$
\left\|\nabla_{\underline{e}_{1}, \ldots, \underline{e}_{k}}^{j} A_{\varepsilon_{n}, m=1}^{a}\left(L^{0}\right) f\right\|_{L_{\infty}\left(\left(\varepsilon_{n} \mathbf{Z}\right)^{d}\right)} \leqslant C_{k, L}(1+a)^{-\frac{1}{2}}\|f\|_{L^{\infty}\left(\left(\varepsilon_{n} \mathbf{Z}\right)^{d}\right)}
$$

noting that (5.4) applies to any translate $x+\Omega_{\varepsilon_{n}}$ of $\Omega_{\varepsilon_{n}}$.

Lemma 5.4. For every integer $k \geqslant 1$, and every $n \geqslant 1, \exists$ a constant $c_{k, L}$ independent of $\varepsilon_{n}$ such that,

$$
\left|\hat{A}_{\varepsilon_{n}, m=1}^{a}\left(L^{0}\right)(p)\right| \leqslant c_{k, L}(1+a)^{-\frac{1}{2}}\left(-\hat{\Delta}_{\varepsilon_{n}}(p)+1\right)^{-k}
$$

Proof. This is the essentially the standard proof that the Fourier transform of a smooth function of compact support has rapid decay. Let

$$
h(x, p)=\int_{\left(\varepsilon_{n} \mathbf{Z}\right)^{n}} A_{e_{n}, m=1}^{a}\left(L^{0}\right)(x, d u) e^{-i p u}
$$

Then

$$
\left(-\hat{\Delta}_{\varepsilon_{n}}(p)+1\right)^{k} h(x, p)=\int_{\left(\varepsilon_{n} Z\right)^{n}} A_{e_{n}, m=1}^{a}\left(L^{0}\right)(x, d u)\left(-\Delta_{u, \varepsilon_{n}}+1\right)^{k} e^{-i p u}
$$


where the $u$ subscript on $\Delta_{u, \varepsilon_{n}}$ indicates the variable it differentiates. Since $\Delta_{u, \varepsilon_{n}}$ is a linear combination of lattice translations under which the lattice is invariant, the exact analogue of integration by parts is valid and we continue with

$$
=\int_{\left(\varepsilon_{n} Z\right)^{n}}\left(-\Delta_{u, \varepsilon_{n}}+1\right)^{k} A_{\varepsilon_{n}, m=1}^{a}\left(L^{0}\right)(x, d u) e^{-i p u}
$$

By the translation invariance (see (3.10)) of $A_{\varepsilon_{n}, m=1}^{a}\left(L^{0}\right)(x, d u)$ we can change the derivatives to $x$

$$
=\int_{\left(\varepsilon_{n} Z\right)^{n}}\left(-\Delta_{x, \varepsilon_{n}}+1\right)^{k} A_{\varepsilon_{n}, m=1}^{a}\left(L^{0}\right)(x, d u) e^{-i p u}
$$

By (5.5) in Corollary 5.3 with $f(u)=\exp (i p . u)$,

$$
\left|\int_{\left(\varepsilon_{n} \mathbf{Z}\right)^{n}}\left(-\Delta_{x, \varepsilon_{n}}+1\right)^{k} A_{\varepsilon_{n}, m=1}^{a}\left(L^{0}\right)(x, d u) e^{-i p u}\right| \leqslant c_{k, L}(1+a)^{-\frac{1}{2}}
$$

Collecting these relations we have

$$
\left|\left(-\hat{\Delta}_{\varepsilon_{n}}(p)+1\right)^{k} h(x, p)\right| \leqslant c_{k, L}(1+a)^{-\frac{1}{2}}
$$

By setting $x=0$ we finish the proof.

Fourier transforms are naturally defined on the Brillouin zone

$$
B_{\varepsilon}=[-\pi / \varepsilon, \pi / \varepsilon]^{d}
$$

There is a constant $c$ independent of $\varepsilon$ such that

$$
p^{2} \geqslant-\hat{\Delta}_{\varepsilon}(p) \geqslant c p^{2} \quad \text { for } \quad p \in B_{\varepsilon}
$$

which follows from $t^{2} / 2 \geqslant 1-\cos t \geqslant c t^{2}$ on $[-\pi, \pi]$.

Theorem 5.5. $\forall n \geqslant 0$ and $\forall k \geqslant 0, \exists$ a constant $c_{k, L}$ independent of $n$ such that

$$
\begin{aligned}
\left|\hat{\Gamma}_{n}^{a}(p)\right| & \leqslant c_{k, L}(1+a)^{-1}\left(1+p^{2}\right)^{-2 k} \quad \text { for } \quad p \in B_{\varepsilon_{n}} \\
\left\|\Gamma_{n}^{a}\right\|_{H_{k}\left(\left(\varepsilon_{n} Z\right)^{d}\right)} & \leqslant c_{k, L}(1+a)^{-1}
\end{aligned}
$$

Proof. From (2.10) and (5.9),

$$
0 \leqslant \hat{G}_{\varepsilon_{n}}^{a}(p) \leqslant\left(-\hat{\Delta}_{\varepsilon_{n}}\right)^{-1} \leqslant \frac{1}{c p_{t_{a}}^{2}}
$$


Combining this with (3.16) and the continuity assertion of Lemma 3.1 we have

$$
\hat{\Gamma}_{\varepsilon_{n}}^{a}(p) \leqslant c_{L}\left(1+p^{2}\right)^{-1}
$$

Case $n=0$. It is sufficient to prove that $\hat{\Gamma}_{\varepsilon_{0}}^{a}(p)$ is bounded by $C(1+a)^{-1}$ with $C$ uniform in $p$ because $B_{\epsilon_{0}}$ is bounded. Referring to $(3.16,2.10)$ we find that

$$
\left|\hat{\Gamma}_{\varepsilon_{0}}^{a}(p)\right| \leqslant 2 \frac{\left|1-\hat{A}_{\varepsilon_{0}}^{a}(p)\right|}{a-\hat{\Delta}_{\varepsilon_{0}}(p)} \leqslant 2 \frac{\left|1-\hat{A}_{\varepsilon_{0}}^{a}(0)\right|}{a}+2 \frac{\left|\hat{A}_{\varepsilon_{0}}^{a}(0)-\hat{A}_{\varepsilon_{0}}^{a}(p)\right|}{a-\hat{\Delta}_{\varepsilon_{0}}(p)}
$$

The first term is continuous at $a=0$ by Corollary 4.2 . Therefore it is bounded by $C(1+a)^{-1}$. The second term is bounded by $C(1+a)^{-1}$ using the same argument (existence of moments of the Poisson measure) as in the proof of Lemma 3.1 .

Case $n \geqslant 1$. By Lemma 5.4, and the bound $\left|\hat{A}_{\varepsilon_{n}, m}^{a}(p)\right| \leqslant 1$ which we use for $m \geqslant 2$

$$
\left|\hat{\mathscr{A}}_{n}^{a}(p)\right|^{2} \leqslant C_{k, L}(1+a)^{-1}\left(p^{2}+1\right)^{-k}
$$

Using these estimates in (3.29) we obtain (5.10).

Proof of (5.11). We have the easily established bound

$$
\left|p^{2}+\hat{\Delta}_{\varepsilon_{n}}(p)\right| \leqslant O(1) \varepsilon_{n}^{2}|p|^{4}
$$

so that

$$
0 \leqslant 1-\hat{\Delta}_{\varepsilon_{n}}(p) \leqslant\left(1+p^{2}\right)\left(1+O(1) \varepsilon_{n}^{2} p^{2}\right)
$$

and hence for any $m \geqslant 0$

$$
\left(1-\hat{\Delta}_{\varepsilon_{n}}(p)\right)^{m}\left|\hat{\Gamma}_{n}^{a}(p)\right|^{2} \leqslant O(1)\left(1+p^{2}\right)^{2 m}\left|\hat{\Gamma}_{n}^{a}(p)\right|^{2} \leqslant c_{k, q, L}(1+a)^{-1}\left(1+p^{2}\right)^{-q}
$$

for any $q \geqslant 0$ by choosing $k$ in (5.10) sufficiently large. Taking $q>d$ proves the theorem because

$$
\left\|\Gamma_{n}^{a}\right\|_{H_{m}\left(\left(\varepsilon_{n} \mathrm{Z}\right)^{d}\right)}^{2} \leqslant \int_{\left[-\frac{\pi}{\varepsilon_{n}}, \frac{\pi}{\varepsilon_{n}}\right]^{d}} \frac{d^{d} p}{(2 \pi)^{d}}\left(1-\hat{\Delta}_{\varepsilon_{n}}(p)\right)^{m}\left|\hat{\Gamma}_{n}^{a}(p)\right|^{2}
$$

Now turn to the Lévy fluctuation covariance given in (4.2). Using the bounds provided in Theorem 5.5 we get 
Corollary 5.6. For $0<\alpha<2$, all $k=0,1, \ldots$, and all $n \geqslant 0$,

$$
\left\|\Gamma_{n}\right\|_{H_{k}\left(\left(\varepsilon_{n} \mathrm{Z}\right)^{d}\right)} \leqslant c_{k, L}
$$

where the constant on the right hand side is independent of $n$.

\section{CONVERGENCE}

Theorem 5.5 and Corollary 5.6 provide uniform bounds in Sobolev norms for fluctuation and block covariances. In particular they are uniform in the lattice spacing $\varepsilon_{n}$. We will now prove that these sequences converge to their formal continuum limits. Continuum objects have the subscript $c$ in place of $\varepsilon$. Thus, as in (2.9),

$$
\begin{aligned}
G_{c}^{a}(x-y) & =\int_{\mathbf{R}^{d}} \frac{d^{d} p}{(2 \pi)^{d}} e^{i p \cdot(x-y)} \hat{G}_{c}^{a}(p) \\
\hat{G}_{c}^{a}(p) & =\left(a+p^{2}\right)^{-1}
\end{aligned}
$$

Recall from Section 3 that

$$
U(R) \equiv U_{c}(R)=\left(-\frac{R}{2}, \frac{R}{2}\right)^{d} \subset \mathbf{R}^{d}
$$

represents an open cube of edge length $R$. In analogy to (3.23) with $c_{c}:=1$ we define the continuum average $A_{c, m}^{a}\left(R_{m}\right)(x, d u)$ by

$$
\int_{\mathbf{R}^{d}} d u A_{c, m}^{a}\left(R_{m}\right)(x, d u) f(u)=\int_{\mathbf{R}^{d}} d z c_{c} g_{m}(x-z) \mathscr{P}_{c, U\left(R_{m}, z\right)}^{a}(x, f)
$$

where

$$
\mathscr{P}_{U_{c}(R, z)}^{a}(x, f)=\int_{\partial U_{c}(R, z)} d u \mathscr{P}_{U_{c}(R, z)}^{a}(x, u) f(u)
$$

is the solution $h_{c}^{a}(x)$ to the continuum Dirichlet problem

$$
\begin{aligned}
\left(-\Delta_{c}+a\right) h_{c}^{a}(x) & =0: x \in U_{c}(R) \\
h_{c}^{a}(x) & =f(x): x \in \partial U_{c}(R)
\end{aligned}
$$

With these notations, the Fourier transform of the continuum analogue of (3.13) is

$$
\hat{\Gamma}_{c}^{a}(p)=G_{c}^{a}(p)-\left|\hat{A}_{c, 0}^{a}\left(R_{0}\right)(p)\right|^{2} \hat{G}_{c}^{a}(p)
$$


and that of (3.29) is

$$
\hat{\Gamma}_{c, n}^{a}=\prod_{m=1}^{n}\left|\hat{A}_{c, m}^{a}\left(R_{m}\right)\right|^{2} \hat{\Gamma}_{c}^{a}
$$

The Lemmas, Propositions, Theorems and their Corollaries of Sections 3,4 and 5 remain true in the continuum with the following caveat: in the continuum the uniform Sobolev bounds of Theorem 5.5 and Corollary 5.6 hold only for $n \geqslant 1$. Aside from this caveat their proofs are identical and need no repetition. When referring to them for the continuum objects we shall simply mention them as the continuum analogues of the relevant results for the lattice.

Recall that $B_{\varepsilon_{n}}$ is the Brillouin zone, defined in (5.8). The main result is

Theorem 6.1. For every integer $k \geqslant 0$,

$$
\Gamma_{c, n}^{a} \rightarrow \Gamma_{c, *}^{a}
$$

in $H_{k}\left(\mathbf{R}^{d}\right)$. Moreover, for every fixed lattice $\left(\varepsilon_{l} \mathbf{Z}\right)^{d}, 0 \leqslant l \leqslant n$ the restriction of $\Gamma_{n}^{a}(x)$ to $\left(\varepsilon_{l} \mathbf{Z}\right)^{d}$ converges to the continuum $\Gamma_{c, *}^{a}(x)$ restricted to $\left(\varepsilon_{l} \mathbf{Z}\right)^{d}$ in the Sobolev norm

$$
\left\|\Gamma_{c, *}^{a}-\Gamma_{n}^{a}\right\|_{H_{k}\left(\left(\varepsilon_{l} \mathbf{Z}\right)^{d}\right)} \rightarrow 0 \quad \text { as } \quad n \rightarrow \infty
$$

Moreover multiple lattice derivatives of $\Gamma_{n}^{a}$ converge to the corresponding continuum derivatives of $\Gamma_{c, *}^{a}$ in the $L_{\infty}\left(\left(\varepsilon_{l} \mathbf{Z}\right)^{d}\right)$ norm.

For the Lévy finite range decomposition (4.2) we apply the last theorem to (4.3) and obtain

Corollary 6.2. For all $k \geqslant 0$,

$$
\left\|\Gamma_{c, *}-\Gamma_{n}\right\|_{H_{k}\left(\left(\varepsilon_{l} \mathbf{Z}\right)^{d}\right)} \rightarrow 0 \quad \text { as } \quad n \rightarrow \infty
$$

Moreover multiple lattice derivatives of $\Gamma_{n}$ converge to the corresponding continuum derivatives of $\Gamma_{c, *}$ in the $L_{\infty}\left(\left(\varepsilon_{l} \mathbf{Z}\right)^{d}\right)$ norm.

We now give some Lemmas which will be employed in the proof of Theorem 6.1. In the following lemmas we consider continuum functions $f: \mathbf{R}^{d} \rightarrow \mathbf{R}$ and use the same symbol for the lattice function $f: \mathbf{Z}^{d} \rightarrow \mathbf{R}$ defined by restriction. Continuum and lattice integration are to be distinguished by the domain of integration. 


\section{Lemma 6.3.}

$$
\|f(\cdot+h)-f(\cdot)\|_{H_{k}\left(\mathbf{R}^{d}\right)} \leqslant c|h| \cdot\|f\|_{H_{k+1}\left(\mathbf{R}^{d}\right)}
$$

where $|h|$ is the norm in $\mathbf{Z}^{d} \subset \mathbf{R}^{d}$. Moreover if $|h| \leqslant R$ we have

$$
\|f(\cdot+h)-f(\cdot)\|_{H_{k}\left(U_{c}(R)\right)} \leqslant c|h| \cdot\|f\|_{H_{k+1}\left(U_{c}(2 R)\right)}
$$

Proof. See Theorem 3.3 on p. 42 of ref. 1.

\section{Lemma 6.4. Define}

$$
Q=-\Delta_{\varepsilon}-\left(-\Delta_{c}\right)
$$

Then we have for every $k \geqslant 0$,

$$
\|Q f\|_{H_{k}\left(\mathbf{R}^{d}\right)} \leqslant c \varepsilon\|f\|_{H_{k+3}\left(\mathbf{R}^{d}\right)}
$$

where the constant $c$ is independent of $\varepsilon$.

Proof. $\nabla_{\underline{e}, \varepsilon}$ is the forward lattice derivative in $(\varepsilon \mathbf{Z})^{d}$, and $\nabla_{\underline{e}, \varepsilon}^{*}$, its $L^{2}\left((\varepsilon \mathbf{Z})^{d}\right)$ adjoint, the backward lattice derivative. Forward and backward derivatives commute. $\nabla_{\underline{e}, c}$ is the continuum derivative in direction $\underline{e}$ and the adjoint is $\nabla_{\underline{e}, c}^{*}=-\nabla_{\underline{e}, c}$. A calculation shows that

$$
\nabla_{\underline{e}, \varepsilon}^{*} \nabla_{\underline{e}, \varepsilon} f(x)=\int_{0}^{1} d t \int_{0}^{1} d s\left(-\nabla_{\underline{e}, c}^{2}\right) f(x+[t-s] \varepsilon \underline{e})
$$

Therefore

$$
Q f(x)=-\sum_{\underline{\underline{e}}} \int_{0}^{1} d t \int_{0}^{1} d s\left(\nabla_{\underline{e}, c}^{2} f(x+[t-s] \varepsilon \underline{e})-\nabla_{\underline{e}, c}^{2} f(x)\right)
$$

since

$$
-\Delta_{\varepsilon}=\sum_{\underline{e}} \nabla_{\underline{e}, \varepsilon}^{*} \nabla_{\underline{e}, \varepsilon}
$$

the lemma is proved by taking norms under the integrals and using Lemma 6.3 (which gives the factor $\varepsilon$ ).

We can now describe the main idea. Let $f: \partial U_{c}(R) \rightarrow \mathbf{R}$ be a continuum function $f: \mathbf{R}^{d} \rightarrow \mathbf{R}$ restricted to the continuum boundary of $U_{c}(R)$. We need to estimate the difference between the solution $h_{c}^{a}$ to the continuum Dirichlet problem and the solution $h_{\varepsilon_{n}}^{a}$ to the lattice Dirichlet 
problem. This will be done by restricting $h_{c}^{a}$ to the lattice. The restriction also solves a lattice Dirichlet problem, but with a non-zero right hand side involving $Q h_{c}^{a}$, which by Lemma 6.4 is $O\left(\varepsilon_{n}\right)$. Thus we consider

$$
\begin{gathered}
h_{\varepsilon_{n}}^{a}(x)-h_{c}^{a}(x): x \in U_{\varepsilon_{n}}\left(\frac{R}{4}\right) \subset U_{c}\left(\frac{R}{4}\right) \\
h_{\varepsilon_{n}}^{a}(x)-h_{c}^{a}(x)=0: x \in \partial U_{\varepsilon_{n}}(R)
\end{gathered}
$$

in Sobolev norms. The difference satisfies zero boundary conditions because we will arrange that the lattice boundary points $\partial U_{\varepsilon_{n}}(R)$ all lie on the continuum boundary $\partial U_{c}(R)$ and both solutions have boundary values $f$ restricted to $\partial U_{\varepsilon_{n}}(R)$.

The lattice cube

$$
U_{\varepsilon}(R)=U(R) \cap(\varepsilon \mathbf{Z})^{d}
$$

has as its boundary

$$
\partial U_{\varepsilon}(R)=\left\{y \notin U_{\varepsilon}(R):|x-y|=\varepsilon, \text { some } x \in U_{\varepsilon}(R)\right\}
$$

From Section 2 we have $\varepsilon_{n}=L^{-n}, L=2^{p}, p \geqslant 1$. In addition we now choose, in accord with (3.22), $R=R_{m}=L^{-(m-1)}: 0 \leqslant m \leqslant n$. Then not only do we have

$$
U_{\varepsilon_{n}}(R) \subset U_{c}(R):=U(R)
$$

but also

$$
\partial U_{\varepsilon_{n}}(R) \subset \partial U_{c}(R)
$$

This last statement follows from the observation that $\varepsilon_{n}=2^{-n p}$ and $\frac{R_{m}}{2}=2^{-m p+p-1}$ so that for $0 \leqslant m \leqslant n$ we have $\frac{R_{m}}{2} \in \varepsilon_{n} \mathbf{Z} \subset \mathbf{R}$. This means that for $d=1$ the boundary points of $U_{\varepsilon_{n}}\left(R_{m}\right)$ coincide with the boundary points of $U\left(R_{m}\right)$. For $d>1$ we are in cubes and the above reasoning easily generalises to (6.14).

Lemma 6.5. Let

$$
f: \mathbf{R}^{d} \rightarrow \mathbf{R}
$$

Then for

$$
R=L^{-(m-1)}: 0 \leqslant m \leqslant n
$$


we have

$$
\left\|\nabla_{\underline{e}_{1}, \ldots, \underline{e}_{k}}^{k}\left(h_{\varepsilon_{n}}^{a}-h_{c}^{a}\right)\right\|_{L_{\infty}\left(U_{\varepsilon_{n}}\left(\frac{R}{4}\right)\right)} \leqslant c_{L, R, k} \varepsilon_{n}\|f\|_{L^{\infty}\left(\partial U_{c}(R)\right)}
$$

where the constant $c_{L, R, k}$ is independent of $n$.

Proof. See Appendix A.

To proceed further we need a formula. First define a new finite difference derivative that acts on continuum functions by:

$$
\tilde{\nabla}_{\underline{e}, \varepsilon} f(z)=\int_{0}^{1} d t(1-t) \nabla_{\underline{e}, c} f(z+t \varepsilon)
$$

Then we have

\section{Lemma 6.6.}

$$
\int_{\mathbf{R}^{d}} d z f(z)-\int_{(\varepsilon \mathbf{Z})^{d}} d z f(z)=\varepsilon \sum_{j=0}^{d-1} \int_{(\varepsilon \mathbf{Z})^{d-j} \times \mathbf{R}^{j}} d z \tilde{\nabla}_{\underline{e}_{j+1}, \varepsilon} f(z)
$$

Proof. We obtain case $d=1$ by

$$
\begin{aligned}
\int_{\mathbf{R}} d z & f(z)-\int_{\varepsilon \mathbf{Z}} d z f(z) \\
= & \sum_{z \in \varepsilon \mathbf{Z}} \int_{[z, z+\varepsilon]} d x f(x)-\int_{\varepsilon \mathbf{Z}} d z f(z) \\
= & \int_{\varepsilon \mathbf{Z}} d z \varepsilon^{-1} \int_{[z, z+\varepsilon]} d x(f(x)-f(z))=\varepsilon \int_{\varepsilon \mathbf{Z}} d z \tilde{\nabla}_{\underline{e}, \varepsilon} f(z)
\end{aligned}
$$

and then the general case is obtained by applying this formula iteratively.

The Fourier transform of $A_{\varepsilon_{n}, m}^{a}\left(R_{m}\right)(0, d u)$ is given by

$$
\hat{A}_{\varepsilon_{n}, m}^{a}\left(R_{m}\right)(p)=c_{\varepsilon_{n-m}} \int_{\left(\varepsilon_{n} Z\right)^{d}} d z g_{m}(z) e^{-i p \cdot z} h_{\varepsilon_{n}, m}^{a}(z, p)
$$

where

$$
h_{\varepsilon_{n}, m}^{a}(z, p)=\int_{\partial U_{\varepsilon_{n}}\left(R_{m}\right)} \mathscr{P}_{U_{\varepsilon_{n}}\left(R_{m}\right)}^{a}(z, d u) e^{i p \cdot u}
$$


Likewise, there is the continuum Fourier transform $\hat{A}_{c, m}^{a}\left(R_{m}\right)(p)$ defined by the same formulas with $\varepsilon_{n}, \varepsilon_{n-m}$ replaced by $c$ and with $c_{c}:=1$.

We wish to estimate the difference $\hat{A}_{c, m+1}^{a}\left(R_{m+1}\right)(p)-\hat{A}_{\varepsilon_{n}, m+1}^{a}\left(R_{m+1}\right)(p)$. This is provided by the following Lemma:

Lemma 6.7. For all integers $m: 0 \leqslant m \leqslant n$, and $n \geqslant 1, \exists$ a constant $c_{L, m}$ independent of $n$ such that

$$
\left|\hat{A}_{c, m+1}^{a}\left(R_{m+1}\right)(p)-\hat{A}_{\varepsilon_{n}, m+1}^{a}\left(R_{m+1}\right)(p)\right| \leqslant c_{L, m} \varepsilon_{n}
$$

Proof. It is easy to see using Lemma 6.6 that we can write

$$
\hat{A}_{c, m+1}^{a}\left(R_{m+1}\right)(p)-\hat{A}_{\varepsilon_{n}, m+1}^{a}\left(R_{m+1}\right)(p)=R_{1}(p)+R_{2}(p)+R_{3}(p) .
$$

where

$$
\begin{aligned}
& R_{1}(p)=\left(1-c_{\varepsilon_{n-1-m}}\right) \int_{\left(\varepsilon_{n} \mathbf{Z}\right)^{d}} d z e^{-i p \cdot z} g_{m+1}(z) h_{c, m+1}^{a}(z, p) \\
& R_{2}(p)=c_{\varepsilon_{n-1-m}} \int_{\left(\varepsilon_{n} \mathbf{Z}\right)^{d}} d z e^{-i p \cdot z} g_{m+1}(z)\left(h_{c, m+1}^{a}(z, p)-h_{\varepsilon_{n}, m+1}^{a}(z, p)\right) \\
& R_{3}(p)=\varepsilon_{n} \sum_{j=0}^{d-1} \int_{\left(\varepsilon_{n} \mathbf{Z}\right)^{d-j} \times \mathbf{R}^{j}} d z e^{-i p \cdot z} \tilde{\nabla}_{\underline{e}_{j+1}, \varepsilon_{n}}\left(g_{m+1}(z) h_{c, m+1}^{a}(z, p)\right)
\end{aligned}
$$

We observe that

$$
\left|1-c_{\varepsilon_{n-1-m}}\right| \leqslant c_{L} \varepsilon_{n}
$$

as follows from the definition (3.7), Lemma 6.6, and the fact that $g$ is a smooth function in $\mathbf{R}^{d}$ of compact support. The integral is bounded by $O(1)$.

For the term involving $R_{2}$ we use Lemma 6.5 with $f(u)=\exp (i p . u)$ which produces the small factor $\varepsilon_{n}$ in the bound.

Finally in $R_{3}$ the $O\left(\varepsilon_{n}\right)$ factor is already there. In the integrand $h_{c, m+1}^{a}(z, p)$ and derivatives are $L_{\infty}$ bounded on the support of $g_{m+1}$ by (the continuum versions of) Proposition 5.2 and Lemma B.1, with $f(u)=$ $\exp (i p . u)$.

Proof. (Theorem 6.1) Let $\epsilon>0$ and fix any $p \neq 0$. Fix any $a \geqslant 0$. We will first prove that

$$
\left|\hat{\Gamma}_{c, *}^{a}(p)-\hat{\Gamma}_{n}^{a}(p)\right|<\epsilon
$$

for all sufficiently large $n$. 
Recall that $\hat{A}_{\varepsilon_{n}, m}^{a}(p)$ and $\hat{A}_{\varepsilon_{n}, m}^{a}(p)$ are Fourier transforms of defective probability measures supported in a cube of side $R_{m}$. Now

$$
\left|1-\hat{A}_{\varepsilon_{n}, m}^{a}(p)\right| \leqslant\left|1-\int A_{\varepsilon_{n}, m}^{a}(0, d u)\right|+\int A_{\varepsilon_{n}, m}^{a}(0, d u)|1-\exp (-i p . u)|
$$

Therefore, by $|1-\exp (-i p . u)| \leqslant c|p . u|$, and Corollary 4.2,

$$
\left|1-\hat{A}_{\varepsilon_{n}, m}^{a}(p)\right| \leqslant c R_{m}|p|+c a R_{m}^{2}
$$

Note that the same bound holds in the continuum, because Corollary 4.2 remains true in the continuum.

From the definition of $\hat{\Gamma}_{c, n}^{a}$ we get

$$
\hat{\Gamma}_{c, n+1}^{a}-\hat{\Gamma}_{c, n}^{a}=\left(\left|\hat{A}_{c, n+1}^{a}\left(R_{n+1}\right)\right|^{2}-1\right) \hat{\Gamma}_{c, n}^{a}
$$

whence, using the continuum analogue of (6.17),

$$
\left|\hat{\Gamma}_{c, n+1}^{a}-\hat{\Gamma}_{c, n}^{a}\right| \leqslant\left(2 c R_{n+1}|p|+2 c a R_{n+1}^{2}\right)\left|\hat{\Gamma}_{c, n}^{a}\right|
$$

Now $\hat{\Gamma}_{c, n}^{a}$ satifies the uniform bound of Theorem 5.5, $R_{n+1}$ decreases geometrically with increasing $n$. Therefore we see from the previous inequality that $\hat{\Gamma}_{c, n}^{a}$ form a Cauchy sequence. This proves the existence of the limit $\hat{\Gamma}_{c, *}^{a}$ satisfying the bound of Theorem 5.5 and the first part of Theorem 6.1 has been proved.

Choose $n$ sufficiently large so that

$$
\left|\hat{\Gamma}_{c, *}^{a}(p)-\hat{\Gamma}_{c, n}^{a}(p)\right|<\frac{\varepsilon}{4}
$$

As shown in the proof of Theorem 5.5, there exists a constant $\gamma$ independent of $\varepsilon_{n}$ such that

$$
\left|\hat{\Gamma}_{\varepsilon_{n}}^{a}(p)\right| \leqslant \gamma
$$

Now (6.17) implies that

$$
|| \hat{A}_{\varepsilon_{n}, m}^{a}|-| \hat{A}_{c, m}^{a}|| \leqslant 2 c R_{m}|p|+2 c a R_{m}^{2}
$$

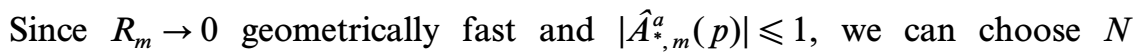
depending on $\epsilon$ such that for all $n>N$,

$$
\left.\left|\prod_{m>N}^{n}\right| \hat{A}_{c, m}^{a}\right|^{2}-\prod_{m>N}^{n}\left|\hat{A}_{\varepsilon_{n}, m}^{a}\right|^{2} \mid<\frac{\epsilon}{4 \gamma}
$$


By Lemma 6.7 there is a constant $C_{N}$ such that

$$
\left.\left|\prod_{m=1}^{N}\right| \hat{A}_{c, m}^{a}\right|^{2}-\prod_{m=1}^{N}\left|\hat{A}_{\varepsilon_{n}, m}^{a}\right|^{2} \mid<C_{N} \varepsilon_{n}<\frac{\epsilon}{4 \gamma}
$$

for all sufficiently large $n$. Finally,

$$
\left|\hat{\Gamma}_{\varepsilon_{n}}^{a}-\hat{\Gamma}_{c}^{a}\right|<\frac{\epsilon}{4}
$$

by the definitions (3.13), the explicit Fourier transforms $(2.10,6.2)$ and Lemma 6.7.

From the definition (3.29) we see that the four inequalities (6.19)-(6.22) imply that

$$
\left|\hat{\Gamma}_{c, n}^{a}-\hat{\Gamma}_{n}^{a}\right|<\frac{3 \epsilon}{4}
$$

(6.23) and (6.18) establish the pointwise convergence

$$
\hat{\Gamma}_{n}^{a}(p) \rightarrow \hat{\Gamma}_{c, *}^{a}(p)
$$

By the dominated convergence theorem using Theorem 5.5 for domination, we have, for any fixed compact set $X \subset \mathbf{R}^{d}$ in momentum space and any $k$

$$
\int_{X} d p\left|\hat{\Gamma}_{c, *}^{a}(p)-\hat{\Gamma}_{n}^{a}(p)\right|^{2}\left(1+p^{2}\right)^{k} \rightarrow 0
$$

as $n \rightarrow \infty$. This proves (6.8) of the theorem, because we can choose $X$ to be a fixed Brillouin zone in the dual of $\left(\varepsilon_{l} \mathbf{Z}\right)^{d}$. The convergence in the $L_{\infty}\left(\left(\varepsilon_{l} \mathbf{Z}\right)^{d}\right)$ norm follows by Sobolev embedding (see Appendix B).

\section{APPENDIX A: LATTICE ELLIPTIC REGULARITY}

Suppose that $h$ solves

$$
\left(a-\Delta_{\varepsilon}\right) h=g
$$

on $(\varepsilon \mathbf{Z})^{d}$ and $\varphi$ has compact support. Then, on $(\varepsilon \mathbf{Z})^{d}$,

$$
\varphi h\left(a-\Delta_{\varepsilon}\right) h=\varphi h g
$$


Integrate over $(\varepsilon \mathbf{Z})^{d}$. By the definition of $-\Delta_{\varepsilon}$ this can be rewritten as

$$
a \int_{(\varepsilon \mathbf{Z})^{d}} d z \varphi h^{2}+\int_{(\varepsilon \mathbf{Z})^{d}} d z \sum_{\underline{e} \in \mathscr{S}}\left(\nabla_{\underline{e}} \varphi h\right)\left(\nabla_{\underline{e}} h\right)=\int_{(\varepsilon \mathbf{Z})^{d}} d z \varphi h g
$$

Surprisingly, calculation shows that this can be rewritten as

$$
a \int_{(\varepsilon \mathbf{Z})^{d}} d z \varphi h^{2}+\frac{1}{2} \sum_{ \pm \underline{e} \in \mathscr{S}} \int_{(\varepsilon \mathbf{Z})^{d}} d z \varphi\left(\nabla_{\underline{e}} h\right)^{2}=\int_{(\varepsilon \mathbf{Z})^{d}} d z\left[\varphi h g+\frac{1}{2}\left(\Delta_{\varepsilon} \varphi\right) h^{2}\right]
$$

In other words with forward and backward derivatives the lattice gives the same formula as the continuum without corrections that go to zero with $\varepsilon$. For $a \geqslant 0$ and for $\varphi \geqslant 0$, (A.3) implies

$$
\begin{aligned}
\frac{1}{2} \sum_{ \pm \underline{e} \in \mathscr{S}} \int_{(\varepsilon \mathbf{Z})^{d}} d z \varphi\left(\nabla_{\underline{e}} h\right)^{2} & \leqslant \int_{(\varepsilon \mathbf{Z})^{d}} d z\left[\varphi h g+\frac{1}{2}\left(\Delta_{\varepsilon} \varphi\right) h^{2}\right] \\
\int_{(\varepsilon \mathbf{Z})^{d}} d z \varphi h^{2} & \leqslant \frac{1}{a} \int_{(\varepsilon \mathbf{Z})^{d}} d z\left[\varphi h g+\frac{1}{2}\left(\Delta_{\varepsilon} \varphi\right) h^{2}\right]
\end{aligned}
$$

Let

$$
[h]_{\varphi, j}^{2}=2^{-j} \sum_{ \pm \underline{e}_{1}, \ldots, \pm \underline{e}_{j} \in \mathscr{S}} \int_{(\varepsilon \mathbf{Z})^{d}} d z\left|\nabla_{\underline{e}_{1}, \ldots, \underline{e}_{j}}^{j} h\right|^{2} \varphi
$$

By applying $j$ finite difference derivatives to (A.1) we find that (A.4) is also true for $h$ and $g$ replaced by derivatives of $h$ and $g$. By the Cauchy-Schwartz inequality on the first term in (A.4),

$$
[h]_{\varphi, j+1}^{2} \leqslant[h]_{\varphi, j}[g]_{\varphi, j}+[h]_{\frac{1}{2} \Delta_{\varepsilon} \varphi, j}^{2}
$$

Simplify the first term using the inequality $a b \leqslant \frac{1}{2}\left(a^{2}+b^{2}\right)$ and use the resulting inequality to iteratively reduce the order of the top derivative in the Sobolev norm,

$$
\|h\|_{\varphi, k}^{2}:=\sum_{j=0}^{k} 2^{-j} \sum_{ \pm \underline{e}_{1}, \ldots, \pm \underline{e}_{j} \in \mathscr{S}} \int_{(\varepsilon \mathbf{Z})^{d}} d z\left|\nabla_{\underline{e}_{1}, \ldots, \underline{e}_{j}}^{j} h\right|^{2} \varphi
$$

We obtain,

$$
\|h\|_{\varphi_{k}, k}^{2} \leqslant\|h\|_{\varphi_{0}, 0}^{2}+\|g\|_{\varphi_{0}, k-1}^{2}
$$


where $\varphi_{0}$ is the final member of a sequence $\varphi_{j}$ of non-negative functions chosen such that

$$
\varphi_{j-1} \geqslant \frac{1}{2} \varphi_{j}+\frac{1}{2} \Delta_{\varepsilon} \varphi_{j}, \quad \varphi_{j-1} \geqslant \varphi_{j}
$$

Proof. (Proposition 5.2). We are given that $h$ solves (A.1) in $U_{\varepsilon}(R)$ with $g=0$. We can estimate the $L_{2}$ norm in $U_{\varepsilon}(R / 2)$ in two different ways. Firstly, for all $a \geqslant 0$ we can use the maximum principle $|h| \leqslant\|f\|_{L^{\infty}\left(\partial U_{\varepsilon}(R)\right)}$

$$
\|h\|_{L_{2}\left(U_{\varepsilon}(R / 2)\right)} \leqslant c R^{d / 2}\|f\|_{L^{\infty}\left(\partial U_{\varepsilon}(R)\right)}
$$

Secondly, for $a \geqslant 1$, we can choose $\varphi$ in (A.5) to be one on $U(R / 2)$ and zero outside $U(R)$ to obtain

$$
\|h\|_{L_{2}\left(U_{\varepsilon}(R / 2)\right)}^{2} \leqslant C_{R} a^{-1}\|h\|_{L_{2}\left(U_{\varepsilon}(R)\right)}^{2}
$$

and then use the maximum principle to bound the right hand side by the $L^{\infty}\left(\partial U_{\varepsilon}(R)\right)$ norm. Therefore, for any smooth $\varphi_{0}$ supported in $U_{\varepsilon}(R / 2)$

$$
\|h\|_{\varphi_{0}, 0}^{2} \leqslant C_{R}(1+a)^{-1}\|f\|_{L^{\infty}\left(\partial U_{\varepsilon}(R)\right)}^{2}
$$

Let $\varphi_{k}, \varphi_{k-1}, \ldots, \varphi_{0} \geqslant 0$ be $C^{\infty}$ continuum functions with compact support in $U(R / 2)$ such that $\varphi_{k}=1$ on $\Omega$ and (A.8) holds. Apply (A.7) and (A.10) to obtain Proposition 5.2.

Remark on Exponential Decay. The correct a dependence is $\exp (-O(\sqrt{a} R))$. We outline how to do this using a method suggested by ref. 2. (A.5), for $g=0$, can be rewritten as

$$
\begin{aligned}
& \int_{(\varepsilon \mathbf{Z})^{d}} d z w h^{2} \leqslant 0 \\
& w:=\left(a-\frac{1}{2} \Delta_{\varepsilon}\right) \varphi
\end{aligned}
$$

Consider the choice $\varphi=\exp (-u)$ with

$$
u(x)=\frac{1}{2} \sqrt{a} \sqrt{1+x^{2}}
$$

Let $\Delta_{0}$ be the continuum Laplacian. The finite difference Laplacian $\Delta_{\varepsilon} \varphi$ can be written as an integral over $\Delta_{0} \varphi$ : for example, in one dimension

$$
\Delta_{\varepsilon} \varphi(x)=\int_{0}^{1} \int_{0}^{1} d s d t \Delta_{0} \varphi(x+[t-s] \varepsilon)
$$


Using this we find $\Delta_{\varepsilon} u \geqslant 0$ and $w \geqslant c(a, \varepsilon) \varphi$ with $c(a, \varepsilon) \approx \exp (-\sqrt{a} \varepsilon)$. Now replace $\exp (-u)$ by $\varphi=\exp (-u) \psi$ where $\psi$ is a smooth, positive, monotonic decreasing function such that $\psi=1$ on $U\left(\frac{2}{3} R\right)$ and vanishes outside $U(R)$. Then $w \geqslant c(a, \varepsilon) \varphi \geqslant 0$ on $U\left(\frac{2}{3} R\right)$ so by taking the part of the integral where $w \neq 0$ to the right hand side of the bound and discarding part of the integral where $w \geqslant 0$ we get

$$
c(a, \varepsilon) \int_{U_{\varepsilon}(R / 2)} d z \varphi h^{2} \leqslant \int_{U_{\varepsilon}(R) \backslash U_{\varepsilon}\left(\frac{2}{3} R\right)} d z(-w) h^{2} \leqslant\|f\|_{L_{\infty}\left(\partial U_{\varepsilon}(R)\right)}^{2} \int_{U_{\varepsilon}(R) \backslash U_{\varepsilon}\left(\frac{2}{3} R\right)} d z|w|
$$

which gives decay because

$$
\left.\varphi\right|_{U(R / 2)} \geqslant\left.\exp (O(\sqrt{a} R)) \varphi\right|_{U(R) \backslash U\left(\frac{2}{3} R\right)}
$$

Preparation for Proof of Lemma 6.5. Suppose (A.1) holds in a domain $U_{\varepsilon}(R)$ and $h$ vanishes on $\partial U_{\varepsilon}(R)$. Then, with $\varphi=1, a \geqslant 0$, (A.2) becomes,

$$
\sum_{\underline{e}} \int_{U_{\varepsilon}(R)} d z\left(\nabla_{\underline{e}} h\right)\left(\nabla_{\underline{e}} h\right) \leqslant \int_{U_{\varepsilon}(R)} d z h g
$$

We estimate the right hand side by the Cauchy-Schwartz inequality and substitute the result into the Poincaré inequality, which is

$$
\|h\|_{L^{2}\left(U_{\epsilon}(R)\right)}^{2} \leqslant C_{R} \sum_{\underline{e}} \int_{U_{\varepsilon}} d z\left(\nabla_{\underline{e}} h\right)\left(\nabla_{\underline{e}} h\right)
$$

where $C_{R}$ is independant of $\varepsilon$. Then

$$
\|h\|_{L^{2}\left(U_{\epsilon}(R)\right)} \leqslant C_{R}\|g\|_{L^{2}\left(U_{\epsilon}(R)\right)}
$$

Returning to (A.7) and using (A.13) we obtain

Lemma A.1. Let $\varphi \geqslant 0$ be a $C^{\infty}$ continuum function with compact support in $U(R)$. By restriction it defines functions on all lattices, denoted by the same letter. Then for $\epsilon$ sufficiently small, there exists a constant $C_{R, \varphi}$ such that the solution to (A.1), with zero boundary conditions on $\partial U_{\varepsilon}(R)$, satisfies

$$
\|h\|_{\varphi, k} \leqslant C_{R, \phi}\|g\|_{H_{k-1}\left(U_{\epsilon}(R)\right)}
$$

where the constant $C_{R, \phi}$ is independant of $\epsilon$. 
Proof. (Lemma 6.5) Write as in Lemma 4.3, $-\Delta_{\varepsilon_{n+1}}=-\Delta_{\varepsilon_{n}}+Q$. Let $h=h_{\varepsilon_{n}}^{a}-h_{\varepsilon_{n+1}}^{a}$. This has zero boundary conditions on $\partial U_{\varepsilon_{n}}(R)$ and

$$
\left(-\Delta_{\varepsilon_{n}}+a\right) h(x)=g(x): x \in U_{\varepsilon_{n}}(R)
$$

where $g=Q h_{\varepsilon_{n+1}}^{a}$. Now apply Lemma A.1 and estimate $g$ by Lemma 6.4 followed by Proposition 5.2. The proof is completed by Sobolev embedding, see Lemma B.1 below, taking $k$ in Lemma A.1 sufficiently large.

\section{APPENDIX B: SOBOLEV SPACES ON THE LATTICE}

Lemma B.1. Let $I^{d}:=[0,1]^{d}, I_{\varepsilon}^{d}=I^{d} \cap(\varepsilon \mathbf{Z})^{d}$ and $X$ be any subset of $I_{\varepsilon}^{d}$. Then

$$
\|u\|_{L^{\infty}(X)} \leqslant C_{d}\|u\|_{H_{d}\left(I_{\varepsilon}^{d}\right)}
$$

Proof. A smooth continuum function $u(x)$, where $x=\left(x_{1}, \ldots, x_{d}\right)$, satisfies

$$
x_{1} \cdots x_{d} u(x)=\int_{0}^{x_{1}} d y_{1} \cdots \int_{0}^{x_{d}} d y_{d} \partial_{1} \cdots \partial_{d}\left(y_{1} \cdots y_{d} u\left(y_{1}, \ldots, y_{d}\right)\right)
$$

and therefore, for $x \in I^{d}:=[0,1]^{d}$,

$$
|u(x)| \leqslant \frac{C_{d}}{\left|x_{1} \cdots x_{d}\right|}\|u\|_{H_{d}\left(I^{d}\right)}
$$

The same proof adapts to the lattice $(\varepsilon \mathbf{Z})^{d}$ with integrals and derivatives being replaced by sums and finite differences so that for $u: I_{\varepsilon}^{d} \rightarrow \mathbf{R}$,

$$
\sup _{x \in J_{\varepsilon}}|u(x)| \leqslant C_{d}\|u\|_{H_{d}\left(I_{\varepsilon}^{d}\right)}
$$

where $J_{\varepsilon}:=[1 / 2,1]_{\varepsilon}^{d}$. For $d=2, I^{2}$ is the union of $[1 / 2,1]^{2},[0,1 / 2]^{2}$, $[1 / 2,1] \times[0,1 / 2]$, and $[0,1 / 2] \times[1 / 2,1]$ and by symmetry the same bound holds with $J_{\varepsilon}$ replaced by any of these boxes. The same argument applies for all dimensions $d$, which implies the lemma.

Lemma B.2 (Poincare Inequality). Let $u: I_{\varepsilon}^{d} \rightarrow \mathbf{R}$ be any function vanishing on the boundary of $I_{\varepsilon}^{d}$. There exists $C$ independent of $\varepsilon$ such that

$$
\int_{I_{\varepsilon}^{d}} d x|u|^{2} \leqslant C\|\nabla u\|_{L^{2}\left(I_{\varepsilon}^{d}\right)}^{2}
$$


Proof. Let $u_{i}(x)$ be the finite difference partial derivative with respect to component $x_{i}$ of $x$. Then, for $i=1, \ldots, d$,

$$
\left|u\left(x_{1}, \ldots, x_{d}\right)\right| \leqslant \int_{I_{\varepsilon}} d x_{i}\left|u_{i}\left(x_{1}, \ldots, x_{d}\right)\right|
$$

The right hand side is a function of all components of $x$ except $x_{i}$. Take the product over $i$ followed by $2 / d$ root or power.

$$
|u(x)|^{2} \leqslant \prod_{i}\left(\int_{I_{\varepsilon}} d x_{i}\left|u_{i}\right|\right)^{\frac{2}{d}} \leqslant \prod_{i}\left(\int_{I_{\varepsilon}} d x_{i}\left|u_{i}\right|^{2}\right)^{\frac{1}{d}}
$$

Integrate both sides over $\left(x_{1}, \ldots, x_{d}\right) \in I_{\varepsilon}^{d}$ and use the Hölder inequality on the right hand side.

$$
\int_{I_{\varepsilon}^{d}} d x|u|^{2} \leqslant \prod_{i}\left(\int_{I_{\varepsilon}^{d}} d x \int_{I_{\varepsilon}} d x_{i}\left|u_{i}\right|^{2}\right)^{\frac{1}{d}} \leqslant \int_{I_{\varepsilon}^{d}} d x \sum_{i}\left|u_{i}\right|^{2}
$$

because the extra integral integrates to unity.

\section{ACKNOWLEDGMENTS}

We thank Gianni Jona-Lasinio for his hospitality in Rome and for many stimulating conversations over the years. P.K.M. thanks Gérard Menessier and André Neveu for fruitful discussions. We thank an anonymous referee for fingering an incorrect Lemma. D.C.B. was supported by NSERC.

\section{REFERENCES}

1. S. Agmon, Lectures on Elliptic Boundary Value Problems, prepared for publication by B. Frank Jones, Jr. with the assistance of George W. Batten, Jr., Van Nostrand Mathematical Studies, No. 2 (Van Nostrand, Princeton, N.J.-Toronto-London, 1965).

2. S. Agmon, Lectures on exponential decay of solutions of second-order elliptic equations: Bounds on eigenfunctions of $N$-body Schrödinger operators, Mathematical Notes, Vol. 29 (Princeton University Press, Princeton, NJ, 1982).

3. Tadeusz Bałaban, (Higgs) ${ }_{2,3}$ quantum fields in a finite volume. I. A lower bound, Comm. Math. Phys. 85:603-626 (1982).

4. Tadeusz Bałaban, (Higgs) $)_{2,3}$ quantum fields in a finite volume II. An upper bound, Comm. Math. Phys. 86:555-594 (1982).

5. D. C Brydges, P. K. Mitter, and B. Scoppola, Critical $\left(\Phi^{4}\right)_{3, \epsilon}$, Comm. Math. Phys. 240:281-327 (2003).

6. J. Fröhlich and T. Spencer, Kosterlitz-Thouless transition in the two dimensional Abelian spin systems and Coulomb gas, Comm. Math. Phys. 81:527 (1981).

7. K. Gawedzki and A. Kupiainen, A rigorous block spin approach to massless lattice theories, Comm. Math. Phys. 77:31-64 (1980). 
8. K. Gawedzki and A. Kupiainen, Block spin renormalization group for dipole gas and $(\nabla \phi)^{4}$, Ann. Phys. 147:198 (1983).

9. K. Gawedzki and A. Kupiainen, Asymptotic freedom beyond perturbation theory, in Critical Phenomena, Random Systems, Gauge Theories, K. Osterwalder and R. Stora, eds. (Les Houches, North Holland, 1986).

10. C. Hainzl and R. Seiringer, General decomposition of radial functions on $\mathbb{R}^{n}$ and applications to $N$-body quantum systems, Lett. Math. Phys. 61:75-84 (2002).

11. P. K. Mitter and B. Scoppola, Renormalization group approach to interacting polymerised manifolds, Comm. Math. Phys. 209:207-261 (2000). 